\title{
FINITE DIFFERENCE/SPECTRAL APPROXIMATIONS FOR THE FRACTIONAL CABLE EQUATION
}

\author{
YUMIN LIN, XIANJUAN LI, AND CHUANJU XU
}

\begin{abstract}
The Cable equation has been one of the most fundamental equations for modeling neuronal dynamics. In this paper, we consider the numerical solution of the fractional Cable equation, which is a generalization of the classical Cable equation by taking into account the anomalous diffusion in the movement of the ions in neuronal system. A schema combining a finite difference approach in the time direction and a spectral method in the space direction is proposed and analyzed. The main contribution of this work is threefold: 1) We construct a finite difference/Legendre spectral schema for discretization of the fractional Cable equation. 2) We give a detailed analysis of the proposed schema by providing some stability and error estimates. Based on this analysis, the convergence of the method is rigourously established. We prove that the overall schema is unconditionally stable, and the numerical solution converges to the exact one with order $O\left(\triangle t^{2-\max \{\alpha, \beta\}}+\triangle t^{-1} N^{-m}\right)$, where $\triangle t, N$ and $m$ are respectively the time step size, polynomial degree, and regularity in the space variable of the exact solution. $\alpha$ and $\beta$ are two different exponents between 0 and 1 involved in the fractional derivatives. 3) Finally, some numerical experiments are carried out to support the theoretical claims.
\end{abstract}

\section{INTRODUCTION}

The key feature in maintaining a voltage difference between a neuron and the external world is the cell membrane which has the property of selectively allowing different ions in and out of the cell. The Nernst-Planck equation of electro-diffusion [16, 18, 29] has been used to describe the movement of ions (e.g. calcium, potassium, sodium, chloride, and magnesium, etc.) in the neuron by taking into account the flux of ions due to pure diffusion and due to ionic drift. However, numerical approximations to the Nernst-Planck equation suffer from several difficulties. First, its numerical solution is expensive due to the multi-dimensionality; Second, it is nontrivial to specify suitable boundary conditions, which are usually unknown in practical applications. In order to overcome these drawbacks, the Cable equation has been introduced to replace the Nernst-Planck equation in some idealized situation. In fact, as indicated in the works [16, 31, if the geometry of the neuron is idealized as a cylinder of length $L$ and diameter $d$ where $d$ is small compared to $L$,

Received by the editor February 7, 2009 and, in revised form, April 11, 2010.

2010 Mathematics Subject Classification. Primary 65M12, 65M06, 65M70, 35S10.

Key words and phrases. Fractional cable equation, numerical solution, stability, convergence.

The research of the first author was partially supported by Fujian NSF under Grant S0750017.

The research of the third author was partially supported by National NSF of China under Grant 10531080, the Excellent Young Teachers Program by the Ministry of Education of China, and 973 High Performance Scientific Computation Research Program 2005CB321703.

(C)2010 American Mathematical Society Reverts to public domain 28 years from publication 
and if the externally applied potential and concentration of ions across the neuron membrane are assumed to be axisymmetric, then the Nernst-Planck equation can be reduced to a one-dimensional Cable equation [15, 18, 30, 31, 35. The wellknown Cable equation can also be derived by directly applying electrical properties to the cell membrane. Roughly, the dendrites of a nervous system can be treated as electrical cables, which consist of continuous pieces of cell membrane. By doing so the dendrites are regarded as a series of simple RC-circuits coupled with an axial resistance, leading to the Cable equation. We will give a brief derivation of this equation in the next section.

Generally speaking, the classical Cable equation is much simpler than the NernstPlanck equation, and there exist a number of methods to efficiently compute its numerical solution. However, in the derivation of this equation, the movement of the ions and their buffering proteins is assumed to be due to standard diffusion. In recent years a wide variety of biological systems have shown anomalous diffusion, and its rates cannot be characterized by a single parameter of the diffusion constant [6. Anomalous diffusion in these biological systems deviates from the standard Fichean description of Brownian motion, the main character of which is that its mean squared displacement is a nonlinear growth with respect to time, such as $\left\langle x^{2}(t)\right\rangle \sim t^{\alpha}$. As examples, single particle tracking experiments have revealed subdiffusion $(0<\alpha<1)$ of proteins and lipids in a variety of cell membranes 6. 10, 11, 12, 37. Anomalous subdiffusion has also been observed in neural cell adhesion molecures [36. Indeed, anomalous diffusion occurs in many other physical situations, such as, transport of fluid in porous media [5], diffusion on liquid surfaces [13, 21, turbulent flow 39, chaotic dynamics charge transport in amorphous semiconductors [33, 34, NMR diffusometry in disordered materials [27, dynamics of a bead in a polymer network [1, and the propagation of mechanical diffusive waves in viscoelastic media [24, etc.

Due to its significant deviation from the dynamics of Brownian motion, the above-mentioned anomalous diffusion in biological systems cannot be adequately described by the traditional Nernst-Planck equation or its simplification, the Cable equation. Very recently, a modified Cable equation was introduced for modeling the anomalous diffusion in spiny neuronal dendrites [14. The resulting governing equation, the so-called fractional Cable equation, is similar to the traditional Cable equation except that the order of derivative with respect to the space and/or time is fractional.

The goal of this paper is to address such an equation, and to design efficient numerical schemes for its numerical solution. It has been known that the feature of the fractional derivatives makes the design of accurate and fast methods difficult. Unlike the integer derivatives, which are local in the sense that the derivative of a function at a certain point in space or time depends only on the function in the vicinity of this point, presence of the integral in the noninteger order derivatives makes the problem global. This means that the solution at a time $t_{k}$ depends on the solutions at all previous time levels $t<t_{k}$. The fact that all previous solutions have to be saved to compute the solution at the current time level would make the storage very expensive if low-order methods are employed for spatial discretization. Thus, it is very desirable to use high-order methods for efficient computations of the numerical solution of this kind of problem. This motivates us to consider the spectral method for spatial discretization since, as compared to a low-order method, 
the spectral method needs fewer grid points to produce highly accurate solutions. In this work, we aim at developing and analyzing a finite difference schema in time and Legendre spectral methods in space for the fractional Cable equation.

Note that some similar investigations have been made for the time fractional diffusion equation. For example, Langlands and Henry [20] considered an implicit numerical schema for a fractional diffusion equation in which the backward Euler approximation is used to discretize the first order time derivative and the L1 schema is used to approximate the fractional order time derivative. Lin and Xu $[23$, proposed a finite difference schema in time and Legendre collocation spectral method in space for the time fractional diffusion equation. Ervin and Roop 9] introduced a variational formulation for the stationary fractional advection dispersion equation. Deng [7] proposed a finite element method for the fractional Fokker-Planck equation.

This work follows the idea proposed in 23 in an attempt to generalize the mixed finite difference/Legendre spectral method in 23 to the fractional Cable equation. The main contribution of this work consists of: (i) constructing a schema based on a finite difference method in time and Legendre spectral method in space for the numerical solution of the fractional Cable equation, which is a generalization of the schema proposed in 23 to the case of multi-fractional derivatives; (ii) carrying out an analysis of stability and convergence of the proposed method. A convergence rate of order $O\left(\triangle t^{2-\max \{\alpha, \beta\}}+\Delta t^{-1} N^{-m}\right)$ is rigourously proved, where $\Delta t$ and $N$ are respectively the time step size and the space resolution, $m$ is the regularity in the space variable of the exact solution. $\alpha$ and $\beta$ are exponents of the fractional derivatives. In particular, an improved technique, as compared to the one used in [23], for the proof of the time error estimate is provided. This new technique allows us to obtain a detailed dependence of the constant that appears in front of $\triangle t^{2-\max \{\alpha, \beta\}}$; (iii) a series of numerical tests, which are conducted to support the theoretical results.

The outline of this paper is as follows. In the next section we first briefly recall the derivation of the fractional Cable equation. Then in section 2 we construct our finite difference/spectral method for this equation. A detailed error analysis is carried out in section 3, to derive the error estimate for the temporal discretization. In section 4, we present a Legendre spectral method for the spatial discretization of the fractional Cable equation. The error estimates are provided for the full discretization problem. Finally, some numerical results are presented in section 5 which support the theoretical statement. Some concluding remarks are given in the final section.

\section{Initial boundary value problem of the fRACtional Cable EQUATion}

To model the information flows in the dendrites of a nervous system, the dendrites are often treated as electrical cables, which consist of continuous pieces of membrane. Then the dendrites can be regarded as a series of simple RC-circuits coupled with an axial resistance that is determined by the properties of the axoplasm. Figure 1 shows a model of a membrane electrical cable broken into small parts, from which we obtain the following equations:

$$
C \partial_{t} V_{i}=-\frac{V_{i}-E}{R}+\frac{V_{i+1}-2 V_{i}+V_{i-1}}{R_{a}},
$$




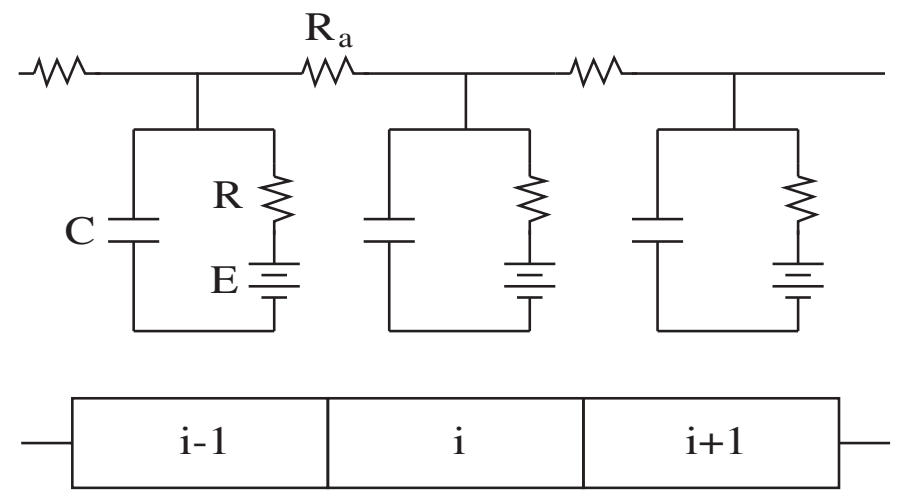

Figure 1. Membrane electrical cable broken into continuous pieces.

where $V_{i}$ is the potential of the membrane piece $i, E$ is the equilibrium potential of the membrane [17, $R_{a}$ is the axial resistance. In (2.1) the piecewise capacitance $C$ is proportional to the area of each piece of membrane, i.e., $C=\pi d l C_{m}$, with $d$ the diameter of the cable, $l$ the length of each piece of membrane, $C_{m}$ a constant independent of the membrane area. Similarly, the piecewise resistance $R$ can be expressed by $R=\frac{R_{m}}{\pi d l}$, with $R_{m}$ a membrane constant. The axial resistance $R_{a}$ is measured by $R_{a}=\frac{l R_{A}}{\pi(d / 2)^{2}}$, with $R_{A}$ an axial material constant, associated to the given cable.

We plug these coefficients into (2.1), let $x_{i}=i l$, define the coordinate along the cable, then we obtain

$$
C_{m} \partial_{t} V\left(x_{i}, t\right)=-\frac{V\left(x_{i}, t\right)-E}{R_{m}}+\frac{d}{4 R_{A}} \frac{V\left(x_{i+1}, t\right)-2 V\left(x_{i}, t\right)+V\left(x_{i-1}, t\right)}{l^{2}},
$$

Taking the limit of the above equation as $l \rightarrow 0$ leads to the continuum Cable equation:

$$
C_{m} \partial_{t} V(x, t)=-\frac{V(x, t)-E}{R_{m}}+\frac{d}{4 R_{A}} \partial_{x}^{2} V(x, t),
$$

where $\partial_{x}^{2}$ stands for $\frac{\partial^{2}}{\partial x^{2}}$.

In order to take into account the anomalous diffusion in the movement of the ions due to, e.g., the heterogeneous nature of the neuronal tissue, or the trapping of ions by buffering proteins [8, 19, 25, 32, 38, 40, the standard Cable equation needs to be modified. As in the derivation of the fractional Fokker-Planck equation 2. 26, taking into account anomalous transport in an external field leads us to consider the following fractional Cable equation:

$$
C_{m} \partial_{t} V(x, t)={ }_{0} D_{t}^{\beta}\left[-\frac{V(x, t)-E}{R_{m}}+\frac{d}{4 R_{A}} \partial_{x}^{2} V(x, t)\right],
$$

where $0<\beta<1,{ }_{0} D_{t}^{\beta}$ denotes the Riemann-Liouville fractional derivative operator defined by

$$
{ }_{0} D_{t}^{\beta} v(x, t)=\frac{1}{\Gamma(1-\beta)} \partial_{t} \int_{0}^{t} \frac{v(x, \tau)}{(t-\tau)^{\beta}} d \tau, \quad 0<\beta<1 .
$$


On the other side, because the neuronal membrane is composed of a different material to the neuron itself [18, we would expect the membrane to have different diffusive properties to the neuron. This consideration leads to introduction of a different fractional exponent $\alpha$ for the anomalous diffusion across the membrane, resulting in a more general fractional Cable equation involving two fractional derivatives:

$$
C_{m} \partial_{t} V(x, t)={ }_{0} D_{t}^{\alpha}\left[-\frac{V(x, t)-E}{R_{m}}\right]+{ }_{0} D_{t}^{\beta}\left[\frac{d}{4 R_{A}} \partial_{x}^{2} V(x, t)\right],
$$

where $0<\alpha<1$. The above Cable equation can be rewritten in a compact form:

$$
\tau_{m} \partial_{t} V={ }_{0} D_{t}^{\alpha}[-V+E]+{ }_{0} D_{t}^{\beta}\left[\nu \partial_{x}^{2} V\right],
$$

where $\tau_{m}=R_{m} C_{m}$ and $\nu=(d / 4) R_{m} / R_{A}$ are two constants: the former is independent of geometry, while the latter depends on the diameter of the cable. The constant $\nu$ determines how quickly the potential decays down the cable.

If we measure the membrane potential with respect to the equilibrium potential, i.e., let $u$ denote $V-E$, and rescale the time and space variables by

$$
t^{*}=\frac{t}{\tau_{m}}, x^{*}=\frac{x \tau_{m}^{\frac{1-\beta}{2}}}{\sqrt{\nu}},
$$

then we arrive at the following fractional Cable equation:

$$
\partial_{t} u=-\mu_{0} D_{t}^{\alpha} u+{ }_{0} D_{t}^{\beta} \partial_{x}^{2} u,
$$

where $\mu=\tau_{m}^{\alpha-1}, 0<\alpha, \beta<1$.

Let $\Lambda=(-1,1)$ be the space domain and $I=(0, T]$ the time domain. We now consider the initial boundary value problem of the fractional Cable equation (2.3) in the domain $\Lambda \times I$, subject to the initial condition:

$$
u(x, 0)=u_{0}(x), \forall x \in \Lambda,
$$

and the boundary condition:

$$
u(-1, t)=u(1, t)=0, \forall t \in I .
$$

Lemma 2.1. The solution $u$ of the problem (2.3) -2.5) satisfies the following energy inequality:

$$
\|u(\cdot, t)\|_{L^{2}(\Lambda)} \leq\left\|u_{0}\right\|_{L^{2}(\Lambda)}, \forall t \in I .
$$

Proof. By multiplying (2.3) by $u$, and integrating the resulting equation in $\Lambda \times(0, t)$, we obtain

$\int_{0}^{t} \frac{d}{d \tau} \int_{\Lambda} \frac{1}{2} u^{2}(x, \tau) d x d \tau=-\mu \int_{0}^{t} \int_{\Lambda}{ }_{0} D_{\tau}^{\alpha} u u d x d \tau+\int_{0}^{t} \int_{\Lambda}{ }_{0} D_{\tau}^{\beta} \partial_{x}^{2} u u d x d \tau, \forall t>0$.

In virtue of Lemma 2.3, Lemma 2.4, and Theorem 2.1 of [22, we have

$\frac{1}{2} \int_{0}^{t} \frac{d}{d \tau}\|u(\cdot, \tau)\|_{L^{2}(\Lambda)}^{2} d \tau+\mu \int_{0}^{t} \int_{\Lambda}\left({ }_{0} D_{\tau}^{\alpha / 2}\right)^{2} d x d \tau+\int_{0}^{t} \int_{\Lambda}\left({ }_{0} D_{\tau}^{\beta / 2} \partial_{x} u\right)^{2} d x d \tau=0$.

This results in

$$
\frac{1}{2} \int_{0}^{t} \frac{d}{d \tau}\|u(\cdot, \tau)\|_{L^{2}(\Lambda)}^{2} d \tau \leq 0, \forall t>0 .
$$

The lemma is proved. 
In order to follow the construction idea used in our previous paper [23, we will use the Caputo fractional derivative instead of the Riemann definition. To this end, we recall the following well-known relation; see e.g. [28]. For $0<\gamma<1$, if $v(t)$ has the integrable first order derivative in $[0, T]$, then

$$
{ }_{0} D_{t}^{\gamma} v(t)=D_{*}^{\gamma} v(t)+\frac{v(0) t^{-\gamma}}{\Gamma(1-\gamma)},
$$

where $D_{*}^{\gamma}$ denotes the Caputo fractional derivative of $\gamma$-order, defined by

$$
D_{*}^{\gamma} v(t)=\frac{1}{\Gamma(1-\gamma)} \int_{0}^{t} \frac{\partial_{\tau} v(\tau)}{(t-\tau)^{\gamma}} d \tau
$$

Then the fractional Cable equation (2.3) can be transformed under the form of Caputo definition:

$$
\partial_{t} u=-\mu D_{*}^{\alpha} u+D_{*}^{\beta} \partial_{x}^{2} u-\frac{\mu}{\Gamma(1-\alpha) t^{\alpha}} u(x, 0)+\frac{1}{\Gamma(1-\beta) t^{\beta}} \partial_{x}^{2} u(x, 0) .
$$

In the next section, we are going to construct and analyze a finite difference schema for the time discretization of the above equation. For ease of notation, we hereafter denote by $c$ a generic constant which may not be the same at different occurrences, but independent of all discretization parameters.

\section{Discretization in time: A Finite DifFEREnCE SCHEMA}

3.1. Construction of the schema. First, we introduce a finite difference schema to discretize the time fractional derivative. For a given integer $K>0$, let $t_{k}=k \triangle t$, $k=0,1, \ldots, K$, where $\Delta t=\frac{T}{K}$ is the time step. By using the Taylor formula with the integral remainder,

$$
f(t)=f(s)+\partial_{t} f(s)(t-s)+\int_{s}^{t} \partial_{\tau}^{2} f(\tau)(t-\tau) d \tau, \forall t, s \in I,
$$

to the function $u(\cdot, t)$ at $t=t_{j}$ and $t=t_{j+1}$ respectively, we obtain

$$
\begin{aligned}
\partial_{s} u(x, s)= & \frac{u\left(x, t_{j+1}\right)-u\left(x, t_{j}\right)}{\Delta t} \\
& -\frac{1}{\Delta t} \int_{s}^{t_{j+1}} \partial_{\tau}^{2} u(x, \tau)\left(t_{j+1}-\tau\right) d \tau+\frac{1}{\Delta t} \int_{s}^{t_{j}} \partial_{\tau}^{2} u(x, \tau)\left(t_{j}-\tau\right) d \tau .
\end{aligned}
$$

Thus for all $0 \leq k \leq K-1$, we have

$$
\begin{aligned}
D_{*}^{\alpha} u & \left(x, t_{k+1}\right)=\frac{1}{\Gamma(1-\alpha)} \sum_{j=0}^{k} \int_{t_{j}}^{t_{j+1}} \partial_{s} u(x, s) \frac{d s}{\left(t_{k+1}-s\right)^{\alpha}} \\
& =\frac{1}{\Gamma(1-\alpha)} \sum_{j=0}^{k} \frac{u\left(x, t_{j+1}\right)-u\left(x, t_{j}\right)}{\Delta t} \int_{t_{j}}^{t_{j+1}} \frac{d s}{\left(t_{k+1}-s\right)^{\alpha}}+r_{\alpha}^{k+1} \\
& =\frac{1}{\Gamma(2-\alpha)} \sum_{j=0}^{k} \frac{u\left(x, t_{k+1-j}\right)-u\left(x, t_{k-j}\right)}{\triangle t^{\alpha}}\left[(j+1)^{1-\alpha}-j^{1-\alpha}\right]+r_{\alpha}^{k+1} \\
& =\frac{1}{\Gamma(2-\alpha)} \sum_{j=0}^{k} a_{j} \frac{u\left(x, t_{k+1-j}\right)-u\left(x, t_{k-j}\right)}{\triangle t^{\alpha}}+r_{\alpha}^{k+1},
\end{aligned}
$$


where

$$
a_{j}=(j+1)^{1-\alpha}-j^{1-\alpha}
$$

and

$$
\begin{aligned}
r_{\alpha}^{k+1}= & \frac{1}{\Gamma(1-\alpha) \Delta t} \sum_{j=0}^{k}\left[-\int_{t_{j}}^{t_{j+1}} \int_{s}^{t_{j+1}} \partial_{\tau}^{2} u(x, \tau) \frac{\left(t_{j+1}-\tau\right)}{\left(t_{k+1}-s\right)^{\alpha}} d \tau d s\right. \\
& \left.+\int_{t_{j}}^{t_{j+1}} \int_{s}^{t_{j}} \partial_{\tau}^{2} u(x, \tau) \frac{\left(t_{j}-\tau\right)}{\left(t_{k+1}-s\right)^{\alpha}} d \tau d s\right] .
\end{aligned}
$$

It will be proved in the Appendix that the following estimate holds:

$$
r_{\alpha}^{k+1} \leq c \triangle t^{2-\alpha}
$$

where $c$ depends only on $M$, a constant measuring $\partial_{t}^{2} u$.

We can derive an expression similar to (3.1) for the fractional derivative term of order $\beta$ in (2.7):

$$
\begin{aligned}
& D_{*}^{\beta} \partial_{x}^{2} u\left(x, t_{k+1}\right) \\
& \quad=\frac{1}{\Gamma(2-\beta)} \sum_{j=0}^{k} \frac{b_{j}}{\triangle t^{\beta}}\left(\partial_{x}^{2} u\left(x, t_{k+1-j}\right)-\partial_{x}^{2} u\left(x, t_{k-j}\right)\right)+r_{\beta}^{k+1},
\end{aligned}
$$

where

$$
b_{j}=(j+1)^{1-\beta}-j^{1-\beta}, j=0,1,2, \ldots, k,
$$

$r_{\beta}^{k+1}$ is the truncation error term having the following estimate:

$$
r_{\beta}^{k+1} \leq c \Delta t^{2-\beta}
$$

with $c$ a constant dependent only on $\partial_{t}^{2} \partial_{x}^{2} u$.

For the discretization of the first-order time derivative $\partial_{t} u$, we use the following development: for $k \geq 1$,

$$
\partial_{t} u\left(x, t_{k+1}\right)=\frac{3 u\left(x, t_{k+1}\right)-4 u\left(x, t_{k}\right)+u\left(x, t_{k-1}\right)}{2 \Delta t}+O\left(\Delta t^{2}\right),
$$

and, for $k=0$,

$$
\partial_{t} u\left(x, t_{1}\right)=\frac{u\left(x, t_{1}\right)-u\left(x, t_{0}\right)}{\Delta t}+O(\Delta t)
$$

For a mesh function $\left\{f^{k}\right\}_{k=0}^{K}$, we define the fractional difference operators $L_{t}^{\alpha}$ by

$$
L_{t}^{\alpha} f^{k+1}=\frac{1}{\Gamma(2-\alpha)} \sum_{j=0}^{k} a_{j} \frac{f^{k+1-j}-f^{k-j}}{\Delta t^{\alpha}}, k \geq 0
$$

and $L_{t}^{\beta}$ by

$$
L_{t}^{\beta} f^{k+1}=\frac{1}{\Gamma(2-\beta)} \sum_{j=0}^{k} b_{j} \frac{f^{k+1-j}-f^{k-j}}{\triangle t^{\beta}}, k \geq 0
$$


We also define the difference operator $L_{t}^{1}$ by

$$
L_{t}^{1} f^{k+1}=\left\{\begin{array}{l}
\frac{3 f^{k+1}-4 f^{k}+f^{k-1}}{2 \Delta t}, k \geq 1, \\
\frac{f^{1}-f^{0}}{\Delta t}, k=0 .
\end{array}\right.
$$

Then by combining (2.7), (3.1), (3.5), (3.8) and (3.9), we have

$$
\begin{aligned}
L_{t}^{1} u(x, & \left.t_{k+1}\right)-r^{k+1} \\
= & -\mu L_{t}^{\alpha} u\left(x, t_{k+1}\right)+L_{t}^{\beta} \partial_{x}^{2} u\left(x, t_{k+1}\right)-\mu r_{\alpha}^{k+1}+r_{\beta}^{k+1} \\
& -\frac{\mu}{\Gamma(1-\alpha)(k+1)^{\alpha} \Delta t^{\alpha}} u(x, 0) \\
& +\frac{1}{\Gamma(1-\beta)(k+1)^{\beta} \Delta t^{\beta}} \partial_{x}^{2} u(x, 0), \quad k \geq 0,
\end{aligned}
$$

where, according to (3.8) and (3.9), $r^{k+1}=O\left(\Delta t^{2}\right)$ for $k \geq 1$, and $r^{k+1}=O(\Delta t)$ for $k=0$.

The above expression motivates us to propose the following finite difference schema for the time discretization of (2.7):

$$
\begin{aligned}
L_{t}^{1} u^{k+1}= & -\mu L_{t}^{\alpha} u^{k+1}+L_{t}^{\beta} \partial_{x}^{2} u^{k+1} \\
& -\frac{\mu}{\Gamma(1-\alpha)(k+1)^{\alpha} \Delta t^{\alpha}} u^{0} \\
& +\frac{1}{\Gamma(1-\beta)(k+1)^{\beta} \Delta t^{\beta}} \partial_{x}^{2} u^{0}, \quad k \geq 0 .
\end{aligned}
$$

In (3.14), $u^{k}$, a simplified notation of $u^{k}(x)$, is an approximation to $u\left(x, t_{k}\right)$. Formally, (3.14) is a schema with the truncation error $r^{k+1}+r_{\alpha}^{k+1}+r_{\beta}^{k+1}$, stemming from the discretizations of the first-order time derivative and the time fractional derivatives of orders $\alpha$ and $\beta$.

In details, the schema (3.14) reads

$$
\begin{aligned}
\frac{3 u^{k+1}-4 u^{k}+u^{k-1}}{2 \Delta t} & =\frac{-\mu}{\Gamma(2-\alpha) \Delta t^{\alpha}}\left(u^{k+1}-\sum_{j=0}^{k-1}\left(a_{j}-a_{j+1}\right) u^{k-j}-a_{k} u^{0}\right) \\
& +\frac{1}{\Gamma(2-\beta) \Delta t^{\beta}}\left(\partial_{x}^{2} u^{k+1}-\sum_{j=0}^{k-1}\left(b_{j}-b_{j+1}\right) \partial_{x}^{2} u^{k-j}-b_{k} \partial_{x}^{2} u^{0}\right) \\
& -\frac{\mu}{\Gamma(1-\alpha)(k+1)^{\alpha} \Delta t^{\alpha}} u^{0}+\frac{1}{\Gamma(1-\beta)(k+1)^{\beta} \Delta t^{\beta}} \partial_{x}^{2} u^{0}, \quad k \geq 1 .
\end{aligned}
$$

For the first step, it is

$$
\begin{aligned}
\frac{u^{1}-u^{0}}{\Delta t}= & \frac{-\mu}{\Gamma(2-\alpha) \Delta t^{\alpha}}\left(u^{1}-u^{0}\right)+\frac{1}{\Gamma(2-\beta) \Delta t^{\beta}}\left(\partial_{x}^{2} u^{1}-\partial_{x}^{2} u^{0}\right) \\
& -\frac{\mu}{\Gamma(1-\alpha) \Delta t^{\alpha}} u^{0}+\frac{1}{\Gamma(1-\beta) \Delta t^{\beta}} \partial_{x}^{2} u^{0} .
\end{aligned}
$$

The equations (3.15) and (3.16), together with the boundary conditions

$$
u^{k+1}(1)=u^{k+1}(-1)=0, \quad k \geq 0,
$$


and the initial condition

$$
u^{0}(x)=u_{0}, \quad x \in \Lambda,
$$

form a complete set of the semi-discretized problems.

3.2. Time error analysis. In this subsection, we aim at carrying out a rigorous error analysis for the time schema (3.15)-(3.16). The error analysis is based on the weak formulation of the semi-discretized problems. To this end, we need some functional spaces that will be used hereafter to define the variational formulation:

$$
\begin{aligned}
H^{1}(\Lambda) & =\left\{v \in L^{2}(\Lambda), \partial_{x} v \in L^{2}(\Lambda)\right\}, \\
H_{0}^{1}(\Lambda) & =\left\{v \in H^{1}(\Lambda),\left.v\right|_{\partial \Lambda}=0\right\}, \\
H^{m}(\Lambda) & =\left\{v \in L^{2}(\Lambda), \partial_{x}^{k} \in L^{2}(\Lambda) \text { for all positive integer } k \leq m\right\},
\end{aligned}
$$

where $L^{2}(\Lambda)$ is the space of measurable functions whose square is Lebesgue integrable in $\Lambda$. The inner products of $L^{2}(\Lambda)$ and $H^{1}(\Lambda)$ are defined respectively by

$$
(u, v)=\int_{\Lambda} u v d x, \quad(u, v)_{1}=(u, v)+\frac{\tilde{\alpha}}{2}(u, v)+\frac{\tilde{\beta}}{2}\left(\partial_{x} u, \partial_{x} v\right)
$$

where

$$
\tilde{\alpha}=\frac{4 \mu \Delta t}{\Gamma(2-\alpha) \Delta t^{\alpha}}, \quad \tilde{\beta}=\frac{4 \Delta t}{\Gamma(2-\beta) \Delta t^{\beta}} .
$$

The norms of $L^{2}(\Lambda)$ and $H^{1}(\Lambda)$ are defined respectively by

$$
\|v\|_{0}=(v, v)^{1 / 2}, \quad\|v\|_{1}=(v, v)_{1}^{1 / 2} .
$$

Here we have used an $H^{1}$-norm differing from the standard one. As we will see later, this $H^{1}$-norm is more convenient than the standard $H^{1}$-norm for the error analysis, although the two norms are equivalent for fixed $\tilde{\alpha}$ and $\tilde{\beta}$.

We now consider the weak formulation of the equation (3.15) subject to the boundary condition (3.17): find $u^{k+1} \in H_{0}^{1}(\Lambda)$, such that for all $v \in H_{0}^{1}(\Lambda)$,

$$
\begin{aligned}
& \left(\frac{3 u^{k+1}-4 u^{k}+u^{k-1}}{2 \Delta t}, v\right) \\
& =-\frac{\mu}{\Gamma(2-\alpha) \Delta t^{\alpha}}\left[\left(u^{k+1}, v\right)-\sum_{j=0}^{k-1}\left(a_{j}-a_{j+1}\right)\left(u^{k-j}, v\right)-a_{k}\left(u^{0}, v\right)\right] \\
& \quad-\frac{1}{\Gamma(2-\beta) \Delta t^{\beta}}\left[\left(\partial_{x} u^{k+1}, \partial_{x} v\right)-\sum_{j=0}^{k-1}\left(b_{j}-b_{j+1}\right)\left(\partial_{x} u^{k-j}, \partial_{x} v\right)-b_{k}\left(\partial_{x} u^{0}, \partial_{x} v\right)\right] \\
& \quad-\frac{\mu}{\Gamma(1-\alpha)(k+1)^{\alpha} \Delta t^{\alpha}}\left(u^{0}, v\right)-\frac{1}{\Gamma(1-\beta)(k+1)^{\beta} \Delta t^{\beta}}\left(\partial_{x} u^{0}, \partial_{x} v\right), \quad k \geq 1 .
\end{aligned}
$$

For the sake of simplification, let's introduce the following notation:

$$
\tilde{\alpha}_{k+1}=\frac{4 \mu \Delta t}{\Gamma(1-\alpha)(k+1)^{\alpha} \Delta t^{\alpha}}, \quad \tilde{\beta}_{k+1}=\frac{4 \Delta t}{\Gamma(1-\beta)(k+1)^{\beta} \Delta t^{\beta}} .
$$


By using the notations (3.19) and (3.22), the schema (3.21) becomes

$$
\begin{aligned}
2\left(3 u^{k+1}-4 u^{k}+u^{k-1}, v\right) \\
=-\tilde{\alpha}\left[\left(u^{k+1}, v\right)-\sum_{j=0}^{k-1}\left(a_{j}-a_{j+1}\right)\left(u^{k-j}, v\right)-a_{k}\left(u^{0}, v\right)\right] \\
\quad-\tilde{\beta}\left[\left(\partial_{x} u^{k+1}, \partial_{x} v\right)-\sum_{j=0}^{k-1}\left(b_{j}-b_{j+1}\right)\left(\partial_{x} u^{k-j}, \partial_{x} v\right)-b_{k}\left(\partial_{x} u^{0}, \partial_{x} v\right)\right] \\
\quad-\tilde{\alpha}_{k+1}\left(u^{0}, v\right)-\tilde{\beta}_{k+1}\left(\partial_{x} u^{0}, \partial_{x} v\right), k \geq 1 .
\end{aligned}
$$

The error estimation will be accomplished with a series of lemmas. We start by giving some properties of $a_{j}$ and $b_{j}$, which can be verified directly.

Lemma 3.1. The coefficients $a_{j}$ and $b_{j}$, defined respectively in (3.2) and (3.6), satisfy

$$
\begin{aligned}
a_{j} & >0, b_{j}>0, j=0,1, \ldots, \\
1 & =a_{0}>a_{1}>\cdots>a_{j}, \quad a_{j} \rightarrow 0 \text { as } j \rightarrow \infty, \\
1 & =b_{0}>b_{1}>\cdots>b_{j}, \quad b_{j} \rightarrow 0 \text { as } j \rightarrow \infty .
\end{aligned}
$$

Lemma 3.2. For the coefficients $\tilde{\alpha}, \tilde{\alpha}_{k+1}, \tilde{\beta}$, and $\tilde{\beta}_{k+1}$, defined respectively in (3.19) and (3.22), we have:

$$
\tilde{\alpha} a_{k+1} \leq \tilde{\alpha}_{k+1} \leq \tilde{\alpha} a_{k}, \quad \tilde{\beta} b_{k+1} \leq \tilde{\beta}_{k+1} \leq \tilde{\beta} b_{k}, \quad \forall k \geq 0 .
$$

Proof. First we prove that $\tilde{\alpha}_{k+1} \leq \tilde{\alpha} a_{k}$. By the definition of $a_{k}$ and the mean-value theorem, we have

$$
\begin{aligned}
a_{k}(k+1)^{\alpha} & =\left((k+1)^{1-\alpha}-k^{1-\alpha}\right)(k+1)^{\alpha} \\
& =(1-\alpha) \xi^{-\alpha}(k+1)^{\alpha}, \quad \text { there exists } \xi \in(k, k+1) \\
& \geq 1-\alpha .
\end{aligned}
$$

Consequently,

$$
\begin{aligned}
\tilde{\alpha} a_{k}-\tilde{\alpha}_{k+1} & =4 \Delta t \mu\left(\frac{a_{k}}{\Gamma(2-\alpha) \Delta t^{\alpha}}-\frac{1}{\Gamma(1-\alpha)(k+1)^{\alpha} \Delta t^{\alpha}}\right) \\
& =4 \Delta t \mu \frac{1}{\Gamma(2-\alpha) \Delta t^{\alpha}(k+1)^{\alpha}}\left(a_{k}(k+1)^{\alpha}-(1-\alpha)\right) \\
& \geq 0 .
\end{aligned}
$$

Next, we prove $\tilde{\alpha} a_{k+1} \leq \tilde{\alpha}_{k+1}$. Similarly, we have

$$
\begin{aligned}
a_{k+1}(k+1)^{\alpha} & =\left((k+2)^{1-\alpha}-(k+1)^{1-\alpha}\right)(k+1)^{\alpha} \\
& =(1-\alpha) \xi^{-\alpha}(k+1)^{\alpha}, \quad \text { there exists } \xi \in(k+1, k+2) \\
& \leq 1-\alpha .
\end{aligned}
$$

Thus

$$
\begin{aligned}
\tilde{\alpha} a_{k+1}-\tilde{\alpha}_{k+1} & =4 \Delta t \mu\left(\frac{a_{k+1}}{\Gamma(2-\alpha) \Delta t^{\alpha}}-\frac{1}{\Gamma(1-\alpha)(k+1)^{\alpha} \Delta t^{\alpha}}\right) \\
& =4 \Delta t \mu \frac{1}{\Gamma(2-\alpha) \Delta t^{\alpha}(k+1)^{\alpha}}\left(a_{k+1}(k+1)^{\alpha}-(1-\alpha)\right) \\
& \leq 0 .
\end{aligned}
$$

Another inequality can be proved in a similar way. 


\section{Lemma 3.3.}

$$
\begin{aligned}
& 2\left(3 u^{k+1}-4 u^{k}+u^{k-1}, u^{k+1}\right) \\
& =\left\|u^{k+1}\right\|_{0}^{2}-\left\|u^{k}\right\|_{0}^{2}+\left\|2 u^{k+1}-u^{k}\right\|_{0}^{2}-\left\|2 u^{k}-u^{k-1}\right\|_{0}^{2} \\
& \quad+\left\|u^{k+1}-2 u^{k}+u^{k-1}\right\|_{0}^{2} .
\end{aligned}
$$

Proof. Direct verification.

The stability result is given in the following theorem.

Theorem 3.1. The semi-discretized problem (3.23) is unconditionally stable in the sense that for all $\triangle t>0$, it holds that

$$
E^{k+1} \leq E^{k}, \quad k=1, \ldots, K-1,
$$

where

$$
E^{k}=\left\|u^{k}\right\|_{0}^{2}+\left\|2 u^{k}-u^{k-1}\right\|_{0}^{2}+\frac{\tilde{\alpha}}{2} \sum_{j=0}^{k} a_{j}\left\|u^{k-j}\right\|_{0}^{2}+\frac{\tilde{\beta}}{2} \sum_{j=0}^{k} b_{j}\left\|\partial_{x} u^{k-j}\right\|_{0}^{2}, \quad k \geq 1 .
$$

On the other hand, the first step schema (3.16) leads to

$$
\begin{gathered}
\left\|u^{1}\right\|_{0}^{2}+\frac{\tilde{\alpha}}{4} \sum_{j=0}^{1} a_{j}\left\|u^{1-j}\right\|_{0}^{2}+\frac{\tilde{\beta}}{4} \sum_{j=0}^{1} b_{j}\left\|\partial_{x} u^{1-j}\right\|_{0}^{2} \\
\leq\left\|u^{0}\right\|_{0}^{2}+\frac{\tilde{\alpha}}{4} a_{0}\left\|u^{0}\right\|_{0}^{2}+\frac{\tilde{\beta}}{4} b_{0}\left\|\partial_{x} u^{0}\right\|_{0}^{2} .
\end{gathered}
$$

Proof. We start by proving (3.25). From (3.16), we have, for all $v \in H_{0}^{1}(\Lambda)$,

$\left(u^{1}-u^{0}, v\right)=-\frac{\tilde{\alpha}}{4}\left(u^{1}-u^{0}, v\right)-\frac{\tilde{\beta}}{4}\left(\partial_{x} u^{1}-\partial_{x} u^{0}, \partial_{x} v\right)-\frac{\tilde{\alpha}_{1}}{4}\left(u^{0}, v\right)-\frac{\tilde{\beta}_{1}}{4}\left(\partial_{x} u^{0}, \partial_{x} v\right)$.

Taking $v=u^{1}$ in the above equality gives

$$
\begin{aligned}
\left(u^{1}, u^{1}\right)= & \left(u^{0}, u^{1}\right)-\frac{\tilde{\alpha}}{4}\left(u^{1}, u^{1}\right)+\frac{1}{4}\left(\tilde{\alpha}-\tilde{\alpha}_{1}\right)\left(u^{0}, u^{1}\right) \\
& -\frac{\tilde{\beta}}{4}\left(\partial_{x} u^{1}, \partial_{x} u^{1}\right)+\frac{1}{4}\left(\tilde{\beta}-\tilde{\beta}_{1}\right)\left(\partial_{x} u^{0}, \partial_{x} u^{1}\right) .
\end{aligned}
$$

By using the triangle inequality and Lemma 3.2. we obtain

$$
\begin{aligned}
2\left\|u^{1}\right\|_{0}^{2} \leq & \left\|u^{0}\right\|_{0}^{2}+\left\|u^{1}\right\|_{0}^{2}-\frac{\tilde{\alpha}}{2}\left\|u^{1}\right\|_{0}^{2}+\frac{1}{4}\left(\tilde{\alpha}-\tilde{\alpha} a_{1}\right)\left\|u^{0}\right\|_{0}^{2}+\frac{1}{4}\left(\tilde{\alpha}-\tilde{\alpha} a_{1}\right)\left\|u^{1}\right\|_{0}^{2} \\
& -\frac{\tilde{\beta}}{2}\left\|\partial_{x} u^{1}\right\|_{0}^{2}+\frac{1}{4}\left(\tilde{\beta}-\tilde{\beta} b_{1}\right)\left\|\partial_{x} u^{0}\right\|_{0}^{2}+\frac{1}{4}\left(\tilde{\beta}-\tilde{\beta} b_{1}\right)\left\|\partial_{x} u^{1}\right\|_{0}^{2} \\
\leq & \left\|u^{0}\right\|_{0}^{2}+\left\|u^{1}\right\|_{0}^{2}-\frac{\tilde{\alpha}}{4}\left(a_{0}\left\|u^{1}\right\|_{0}^{2}+a_{1}\left\|u^{0}\right\|_{0}^{2}\right)-\frac{\tilde{\beta}}{4}\left(b_{0}\left\|\partial_{x} u^{1}\right\|_{0}^{2}+b_{1}\left\|\partial_{x} u^{0}\right\|_{0}^{2}\right) \\
& +\frac{\tilde{\alpha}}{4} a_{0}\left\|u^{0}\right\|_{0}^{2}+\frac{\tilde{\beta}}{4} b_{0}\left\|\partial_{x} u^{0}\right\|_{0}^{2}-\frac{\tilde{\alpha}}{4} a_{1}\left\|u^{1}\right\|_{0}^{2}-\frac{\tilde{\beta}}{4} b_{1}\left\|\partial_{x} u^{1}\right\|_{0}^{2} .
\end{aligned}
$$

In the last inequality we have used the fact that $a_{0}=b_{0}=1$. Then a simple rearrangement yields: 


$$
\begin{aligned}
\left\|u^{1}\right\|_{0}^{2} & +\frac{\tilde{\alpha}}{4} \sum_{j=0}^{1} a_{j}\left\|u^{1-j}\right\|_{0}^{2}+\frac{\tilde{\beta}}{4} \sum_{j=0}^{1} b_{j}\left\|\partial_{x} u^{1-j}\right\|_{0}^{2}+\frac{\tilde{\alpha}}{4} a_{1}\left\|u^{1}\right\|_{0}^{2}+\frac{\tilde{\beta}}{4} b_{1}\left\|\partial_{x} u^{1}\right\|_{0}^{2} \\
\leq & \left\|u^{0}\right\|_{0}^{2}+\frac{\tilde{\alpha}}{4} a_{0}\left\|u^{0}\right\|_{0}^{2}+\frac{\tilde{\beta}}{4} b_{0}\left\|\partial_{x} u^{0}\right\|_{0}^{2} .
\end{aligned}
$$

This proves 3.25) since the last two terms in LHS of the above inequality are positive.

Now we turn to prove (3.24). Taking $v=u^{k+1}$ in (3.23), and using the triangle inequality and Lemmas 3.2 and 3.3 , we have

$$
\begin{aligned}
\left\|u^{k+1}\right\|_{0}^{2} & -\left\|u^{k}\right\|_{0}^{2}+\left\|2 u^{k+1}-u^{k}\right\|_{0}^{2}-\left\|2 u^{k}-u^{k-1}\right\|_{0}^{2} \\
& +\left\|u^{k+1}-2 u^{k}+u^{k-1}\right\|_{0}^{2} \\
\leq & -\tilde{\alpha}\left\|u^{k+1}\right\|_{0}^{2}+\frac{\tilde{\alpha}}{2} \sum_{j=0}^{k-1}\left(a_{j}-a_{j+1}\right)\left(\left\|u^{k-j}\right\|_{0}^{2}+\left\|u^{k+1}\right\|_{0}^{2}\right) \\
& +\frac{1}{2}\left(\tilde{\alpha} a_{k}-\tilde{\alpha}_{k+1}\right)\left(\left\|u^{0}\right\|_{0}^{2}+\left\|u^{k+1}\right\|_{0}^{2}\right) \\
& -\tilde{\beta}\left\|\partial_{x} u^{k+1}\right\|_{0}^{2}+\frac{\tilde{\beta}}{2} \sum_{j=0}^{k-1}\left(b_{j}-b_{j+1}\right)\left(\left\|\partial_{x} u^{k-j}\right\|_{0}^{2}+\left\|\partial_{x} u^{k+1}\right\|_{0}^{2}\right) \\
& +\frac{1}{2}\left(\tilde{\beta} b_{k}-\tilde{\beta}_{k+1}\right)\left(\left\|\partial_{x} u^{0}\right\|_{0}^{2}+\left\|\partial_{x} u^{k+1}\right\|_{0}^{2}\right) \\
\leq & \left.-\tilde{\alpha}+\frac{\tilde{\alpha}}{2} \sum_{j=0}^{k-1}\left(a_{j}-a_{j+1}\right)\right]\left\|u^{k+1}\right\|_{0}^{2}+\frac{\tilde{\alpha}}{2} \sum_{j=0}^{k-1}\left(a_{j}-a_{j+1}\right)\left\|u^{k-j}\right\|_{0}^{2} \\
& +\frac{1}{2}\left(\tilde{\alpha} a_{k}-\tilde{\alpha} a_{k+1}\right)\left\|u^{0}\right\|_{0}^{2}+\frac{1}{2}\left(\tilde{\alpha} a_{k}-\tilde{\alpha}_{k+1}\right)\left\|u^{k+1}\right\|_{0}^{2} \\
& {\left[-\tilde{\beta}+\frac{\tilde{\beta}}{2} \sum_{j=0}^{k-1}\left(b_{j}-b_{j+1}\right)\right]\left\|\partial_{x} u^{k+1}\right\|_{0}^{2}+\frac{\tilde{\beta}}{2} \sum_{j=0}^{k-1}\left(b_{j}-b_{j+1}\right)\left\|\partial_{x} u^{k-j}\right\|_{0}^{2} } \\
& +\frac{1}{2}\left(\tilde{\beta} b_{k}-\tilde{\beta} b_{k+1}\right)\left\|\partial_{x} u^{0}\right\|_{0}^{2}+\frac{1}{2}\left(\tilde{\beta} b_{k}-\tilde{\beta} \tilde{\beta}_{k+1}\right)\left\|\partial_{x} u^{k+1}\right\|_{0}^{2} .
\end{aligned}
$$

Noting that

$$
\sum_{j=0}^{k-1}\left(a_{j}-a_{j+1}\right)=1-a_{k}, \quad \sum_{j=0}^{k-1}\left(b_{j}-b_{j+1}\right)=1-b_{k},
$$

then combining all $\left\|u^{k+1}\right\|_{0}^{2}$ terms in RHS of (3.26) gives

$$
\left[-\tilde{\alpha}+\frac{\tilde{\alpha}}{2} \sum_{j=0}^{k-1}\left(a_{j}-a_{j+1}\right)\right]\left\|u^{k+1}\right\|_{0}^{2}+\frac{\tilde{\alpha}}{2} a_{k}\left\|u^{k+1}\right\|_{0}^{2}=-\frac{\tilde{\alpha}}{2}\left\|u^{k+1}\right\|_{0}^{2},
$$

and combining all $\left\|\partial_{x} u^{k+1}\right\|_{0}^{2}$ terms gives

$$
\left[-\tilde{\beta}+\frac{\tilde{\beta}}{2} \sum_{j=0}^{k-1}\left(b_{j}-b_{j+1}\right)\right]\left\|\partial_{x} u^{k+1}\right\|_{0}^{2}+\frac{\tilde{\beta}}{2} b_{k}\left\|\partial_{x} u^{k+1}\right\|_{0}^{2}=-\frac{\tilde{\beta}}{2}\left\|\partial_{x} u^{k+1}\right\|_{0}^{2} .
$$


Thus (3.26) becomes

$$
\begin{aligned}
\left\|u^{k+1}\right\|_{0}^{2}- & \left\|u^{k}\right\|_{0}^{2}+\left\|2 u^{k+1}-u^{k}\right\|_{0}^{2}-\left\|2 u^{k}-u^{k-1}\right\|_{0}^{2} \\
& +\left\|u^{k+1}-2 u^{k}+u^{k-1}\right\|_{0}^{2} \\
\leq & -\frac{\tilde{\alpha}}{2}\left\|u^{k+1}\right\|_{0}^{2}+\frac{\tilde{\alpha}}{2} \sum_{j=0}^{k-1} a_{j}\left\|u^{k-j}\right\|_{0}^{2}-\frac{\tilde{\alpha}}{2} \sum_{j=1}^{k} a_{j}\left\|u^{k+1-j}\right\|_{0}^{2} \\
& -\frac{\tilde{\alpha}_{k+1}}{2}\left\|u^{k+1}\right\|_{0}^{2}+\frac{\tilde{\alpha}}{2} a_{k}\left\|u^{0}\right\|_{0}^{2}-\frac{\tilde{\alpha}}{2} a_{k+1}\left\|u^{0}\right\|_{0}^{2} \\
& -\frac{\tilde{\beta}}{2}\left\|\partial_{x} u^{k+1}\right\|_{0}^{2}+\frac{\tilde{\beta}}{2} \sum_{j=0}^{k-1} b_{j}\left\|\partial_{x} u^{k-j}\right\|_{0}^{2}-\frac{\tilde{\beta}}{2} \sum_{j=1}^{k} b_{j}\left\|\partial_{x} u^{k+1-j}\right\|_{0}^{2} \\
& -\frac{\tilde{\beta}_{k+1}}{2}\left\|\partial_{x} u^{k+1}\right\|_{0}^{2}+\frac{\tilde{\beta}}{2} b_{k}\left\|\partial_{x} u^{0}\right\|_{0}^{2}-\frac{\tilde{\beta}}{2} b_{k+1}\left\|\partial_{x} u^{0}\right\|_{0}^{2} \\
= & -\frac{\tilde{\alpha}}{2} \sum_{j=0}^{k+1} a_{j}\left\|u^{k+1-j}\right\|_{0}^{2}+\frac{\tilde{\alpha}}{2} \sum_{j=0}^{k} a_{j}\left\|u^{k-j}\right\|_{0}^{2} \\
& -\frac{\tilde{\beta}^{k}}{2} \sum_{j=0}^{k+1} b_{j}\left\|\partial_{x} u^{k+1-j}\right\|_{0}^{2}+\frac{\tilde{\beta}}{2} \sum_{j=0}^{k} b_{j}\left\|\partial_{x} u^{k-j}\right\|_{0}^{2} \\
& -\frac{\tilde{\alpha}_{k+1}}{2}\left\|u^{k+1}\right\|_{0}^{2}-\frac{\tilde{\beta}_{k+1}}{2}\left\|\partial_{x} u^{k+1}\right\|_{0}^{2} .
\end{aligned}
$$

Removing the last term from LHS and the last two terms from RHS of the above inequality, we finally obtain (3.24).

We are now in a position to carry out an error estimation for the solution of the semi-discretized problem. The error estimate is given in the following theorem.

Theorem 3.2. Let $u$ be the solution of the continuous problem (2.3) -(2.5), $\left\{u^{k}\right\}_{k=0}^{K}$ be the time-discrete solution of (3.14) with the initial condition $u^{0}(x)=u(x, 0)$. Then

$$
\left\|u\left(x, t_{k}\right)-u^{k}\right\|_{1} \leq c T^{\frac{1+\alpha}{2}} \Delta t^{\min (2-\alpha, 2-\beta)}, k \geq 1,
$$

where $c$ is independent of $T$ and $\triangle t$.

Proof. From (3.13),$\left\{u\left(x, t_{k}\right)\right\}_{k=0}^{K}$ satisfies

$$
\begin{aligned}
L_{t}^{1} u\left(x, t_{k+1}\right) & \\
= & -\mu L_{t}^{\alpha} u\left(x, t_{k+1}\right)+L_{t}^{\beta} \partial_{x}^{2} u\left(x, t_{k+1}\right)-\frac{\mu u(x, 0)}{\Gamma(1-\alpha)(k+1)^{\alpha} \Delta t^{\alpha}} \\
& +\frac{1}{\Gamma(1-\beta)(k+1)^{\beta} \Delta t^{\beta}} \partial_{x}^{2} u(x, 0)+R^{k+1}, \quad k \geq 0,
\end{aligned}
$$

where $R^{k+1}=r^{k+1}-\mu r_{\alpha}^{k+1}+r_{\beta}^{k+1}$. 
Let $e^{k}(x)=u\left(x, t_{k}\right)-u^{k}(x), k \geq 0$, then substituting (3.28) from (3.14) yields

$$
\begin{aligned}
L_{t}^{1} e^{k+1} & \\
= & -\mu L_{t}^{\alpha} e^{k+1}+L_{t}^{\beta} \partial_{x}^{2} e^{k+1}-\frac{\mu}{\Gamma(1-\alpha)(k+1)^{\alpha} \Delta t^{\alpha}} e^{0} \\
& +\frac{1}{\Gamma(1-\beta)(k+1)^{\beta} \Delta t^{\beta}} \partial_{x}^{2} e^{0}+R^{k+1}, \quad k \geq 0 .
\end{aligned}
$$

Similar to the proof of Theorem 3.1, we have, from (3.29),

$$
\begin{aligned}
\left(e^{1}, e^{1}\right)= & \left(e^{0}, e^{1}\right)-\frac{\tilde{\alpha}}{4}\left(e^{1}, e^{1}\right)+\frac{1}{4}\left(\tilde{\alpha}-\tilde{\alpha}_{1}\right)\left(e^{0}, e^{1}\right) \\
& -\frac{\tilde{\beta}}{4}\left(\partial_{x} e^{1}, \partial_{x} e^{1}\right)+\frac{1}{4}\left(\tilde{\beta}-\tilde{\beta}_{1}\right)\left(\partial_{x} e^{0}, \partial_{x} e^{1}\right)+\triangle t\left(R^{1}, e^{1}\right) .
\end{aligned}
$$

Following the method in Theorem 3.1, we obtain

$$
\begin{aligned}
\left\|e^{1}\right\|_{0}^{2} & +\frac{\tilde{\alpha}}{4} \sum_{j=0}^{1} a_{j}\left\|e^{1-j}\right\|_{0}^{2}+\frac{\tilde{\beta}}{4} \sum_{j=0}^{1} b_{j}\left\|\partial_{x} e^{1-j}\right\|_{0}^{2}+\frac{\tilde{\alpha}}{4} a_{1}\left\|e^{1}\right\|_{0}^{2}+\frac{\tilde{\beta}}{4} b_{1}\left\|\partial_{x} e^{1}\right\|_{0}^{2} \\
& \leq\left\|e^{0}\right\|_{0}^{2}+\frac{\tilde{\alpha}}{4} a_{0}\left\|e^{0}\right\|_{0}^{2}+\frac{\tilde{\beta}}{4} b_{0}\left\|\partial_{x} e^{0}\right\|_{0}^{2}+2 \triangle t\left\|R^{1}\right\|_{0}\left\|e^{1}\right\|_{0} .
\end{aligned}
$$

By using the fact that $e^{0}=0$, we get

$$
\left\|e^{1}\right\|_{0}^{2}+\frac{\tilde{\alpha}}{4} \sum_{j=0}^{1} a_{j}\left\|e^{1-j}\right\|_{0}^{2}+\frac{\tilde{\beta}}{4} \sum_{j=0}^{1} b_{j}\left\|\partial_{x} e^{1-j}\right\|_{0}^{2} \leq 2 \triangle t\left\|R^{1}\right\|_{0}\left\|e^{1}\right\|_{0} .
$$

Then applying Young's inequality to the last term in RHS results in

$$
\frac{1}{2}\left\|e^{1}\right\|_{0}^{2}+\frac{\tilde{\alpha}}{4} \sum_{j=0}^{1} a_{j}\left\|e^{1-j}\right\|_{0}^{2}+\frac{\tilde{\beta}}{4} \sum_{j=0}^{1} b_{j}\left\|\partial_{x} e^{1-j}\right\|_{0}^{2} \leq 2 \triangle t^{2}\left\|R^{1}\right\|_{0}^{2} .
$$

Note again that $a_{0}=b_{0}=1$ and $R^{1}=O(\triangle t)+O\left(\triangle t^{2-\alpha}\right)+O\left(\triangle t^{2-\beta}\right)$, gives us

$$
\left\|e^{1}\right\|_{1} \leq 2 \triangle t\left\|R^{1}\right\|_{0} \leq c \triangle t^{2} .
$$

Thus (3.27) holds for $k=1$. Now we turn to prove (3.27) for $k \geq 2$. By (3.10), (3.11), and (3.12), we get

$$
\begin{aligned}
2\left(3 e^{k+1}\right. & \left.-4 e^{k}+e^{k-1}, v\right) \\
= & -\tilde{\alpha}\left[\left(e^{k+1}, v\right)-\sum_{j=0}^{k-1}\left(a_{j}-a_{j+1}\right)\left(e^{k-j}, v\right)-a_{k}\left(e^{0}, v\right)\right] \\
& -\tilde{\beta}\left[\left(\partial_{x} e^{k+1}, \partial_{x} v\right)-\sum_{j=0}^{k-1}\left(b_{j}-b_{j+1}\right)\left(\partial_{x} e^{k-j}, \partial_{x} v\right)-b_{k}\left(\partial_{x} e^{0}, \partial_{x} v\right)\right] \\
& -\tilde{\alpha}_{k+1}\left(e^{0}, v\right)-\tilde{\beta}_{k+1}\left(\partial_{x} e^{0}, \partial_{x} v\right)+4 \Delta t\left(R^{k+1}, v\right), \quad k \geq 1, \forall v \in H_{0}^{1}(\Lambda) .
\end{aligned}
$$


Taking $v=e^{k+1}$ and following a similar procedure as in Theorem 3.1 we have

$$
\begin{aligned}
\left\|e^{k+1}\right\|_{0}^{2} & +\left\|2 e^{k+1}-e^{k}\right\|_{0}^{2}-\left\|e^{k}\right\|_{0}^{2}-\left\|2 e^{k}-e^{k-1}\right\|_{0}^{2}+\left\|e^{k+1}-2 e^{k}+e^{k-1}\right\|_{0}^{2} \\
\leq & -\frac{\tilde{\alpha}}{2} \sum_{j=0}^{k+1} a_{j}\left\|e^{k+1-j}\right\|_{0}^{2}+\frac{\tilde{\alpha}}{2} \sum_{j=0}^{k} a_{j}\left\|e^{k-j}\right\|_{0}^{2} \\
& -\frac{\tilde{\beta}}{2} \sum_{j=0}^{k+1} b_{j}\left\|\partial_{x} e^{k+1-j}\right\|_{0}^{2}+\frac{\tilde{\beta}}{2} \sum_{j=0}^{k} b_{j}\left\|\partial_{x} e^{k-j}\right\|_{0}^{2} \\
& -\frac{\tilde{\alpha}_{k+1}}{2}\left\|e^{k+1}\right\|_{0}^{2}-\frac{\tilde{\beta}_{k+1}}{2}\left\|\partial_{x} e^{k+1}\right\|_{0}^{2}+4 \Delta t\left|\left(R^{k+1}, e^{k+1}\right)\right| .
\end{aligned}
$$

Let

$$
\varepsilon^{k}:=\left\|e^{k}\right\|_{0}^{2}+\left\|2 e^{k}-e^{k-1}\right\|_{0}^{2}+\frac{\tilde{\alpha}}{2} \sum_{j=0}^{k} a_{j}\left\|e^{k-j}\right\|_{0}^{2}+\frac{\tilde{\beta}}{2} \sum_{j=0}^{k} b_{j}\left\|\partial_{x} e^{k-j}\right\|_{0}^{2},
$$

then

$$
\begin{aligned}
\varepsilon^{k+1} & \leq \varepsilon^{k}-\frac{\tilde{\alpha}_{k+1}}{2}\left\|e^{k+1}\right\|_{0}^{2}-\frac{\tilde{\beta}_{k+1}}{2}\left\|\partial_{x} e^{k+1}\right\|_{0}^{2}+4 \Delta t\left|\left(R^{k+1}, e^{k+1}\right)\right| \\
& \leq \varepsilon^{k}-\frac{\tilde{\alpha}_{k+1}}{2}\left\|e^{k+1}\right\|_{0}^{2}-\frac{\tilde{\beta}_{k+1}}{2}\left\|\partial_{x} e^{k+1}\right\|_{0}^{2}+\frac{8 \Delta t^{2}}{\tilde{\alpha}_{k+1}}\left\|R^{k+1}\right\|_{0}^{2}+\frac{\tilde{\alpha}_{k+1}}{2}\left\|e^{k+1}\right\|_{0}^{2} \\
& \leq \varepsilon^{k}+\frac{8 \Delta t^{2}}{\tilde{\alpha}_{k+1}}\left\|R^{k+1}\right\|_{0}^{2} .
\end{aligned}
$$

Recalling the definition (3.22) of $\tilde{\alpha}_{k+1}$, we have

$$
\frac{8 \Delta t^{2}}{\tilde{\alpha}_{k+1}}=\frac{2 \Gamma(1-\alpha)(k+1)^{\alpha} \Delta t^{\alpha}}{\mu} \Delta t=\frac{2 \Gamma(1-\alpha) t_{k+1}^{\alpha}}{\mu} \Delta t .
$$

Consequently,

$$
\begin{aligned}
\varepsilon^{k+1} & \leq \varepsilon^{k}+\frac{2 \Gamma(1-\alpha) t_{k+1}^{\alpha}}{\mu} \Delta t\left\|R^{k+1}\right\|_{0}^{2} \\
& \leq \varepsilon^{1}+\sum_{j=1}^{k} \frac{2 \Gamma(1-\alpha) t_{j+1}^{\alpha}}{\mu} \Delta t\left\|R^{j+1}\right\|_{0}^{2} \\
& \leq \varepsilon^{1}+\frac{2 \Gamma(1-\alpha) t_{k+1}^{1+\alpha}}{\mu} \max _{2 \leq j \leq k+1}\left\|R^{j}\right\|_{0}^{2} .
\end{aligned}
$$

Taking into account (3.30) and the fact that $\left\|R^{j}\right\|_{0}=O\left(\triangle t^{2}\right)+O\left(\triangle t^{2-\alpha}\right)+$ $O\left(\triangle t^{2-\beta}\right), j \geq 2$, we obtain

$$
\varepsilon^{k} \leq c_{1} \triangle t^{4}+c_{2} T^{1+\alpha}\left(\triangle t^{2-\alpha}+\triangle t^{2-\beta}\right)^{2}, \forall k=1,2, \ldots, K,
$$

where $c_{2}$ depends on $\mu$. Thus

$$
\left\|e^{k}\right\|_{1} \leq c T^{\frac{1+\alpha}{2}}\left(\triangle t^{2-\alpha}+\triangle t^{2-\beta}\right), \forall k=1,2, \ldots, K,
$$

where $c$ is independent of $T$ and $\Delta t$. The proof is completed. 
Remark 3.1. In the case that $\mu$ is small, it may be more interesting to derive an alternative estimate of (3.32). In fact, by using the Poincaré inequality, we have for (3.31):

$$
\begin{aligned}
\varepsilon^{k+1} & \leq \varepsilon^{k}-\frac{\tilde{\alpha}_{k+1}}{2}\left\|e^{k+1}\right\|_{0}^{2}-\frac{\tilde{\beta}_{k+1}}{2}\left\|\partial_{x} e^{k+1}\right\|_{0}^{2}+4 \Delta t\left|\left(R^{k+1}, e^{k+1}\right)\right| \\
& \leq \varepsilon^{k}-\frac{\tilde{\beta}_{k+1}}{2}\left\|\partial_{x} e^{k+1}\right\|_{0}^{2}+4 \Delta t\left\|R^{k+1}\right\|_{0}\left\|e^{k+1}\right\|_{0} \\
& \leq \varepsilon^{k}-\frac{\tilde{\beta}_{k+1}}{2}\left\|\partial_{x} e^{k+1}\right\|_{0}^{2}+4 c_{\Lambda} \Delta t\left\|R^{k+1}\right\|_{0}\left\|\partial_{x} e^{k+1}\right\|_{0}, \text { with } c_{\Lambda} \text { Poincaré constant } \\
& \leq \varepsilon^{k}-\frac{\tilde{\beta}_{k+1}}{2}\left\|\partial_{x} e^{k+1}\right\|_{0}^{2}+\frac{8 c_{\Lambda}^{2} \Delta t^{2}}{\tilde{\beta}_{k+1}}\left\|R^{k+1}\right\|_{0}^{2}+\frac{\tilde{\beta}_{k+1}}{2}\left\|\partial_{x} e^{k+1}\right\|_{0}^{2} \\
& \leq \varepsilon^{k}+\frac{8 c_{\Lambda}^{2} \Delta t^{2}}{\tilde{\beta}_{k+1}}\left\|R^{k+1}\right\|_{0}^{2} .
\end{aligned}
$$

Then by using the definition of $\tilde{\beta}_{k+1}$, i.e. (3.22), we have

$$
\frac{8 \Delta t^{2}}{\tilde{\beta}_{k+1}}=2 \Gamma(1-\beta)(k+1)^{\beta} \Delta t^{\beta} \Delta t=2 \Gamma(1-\beta) t_{k+1}^{\beta} \Delta t .
$$

Following in this way, we will obtain a similar estimate as (3.32), but with $c_{2}$ independent of $\mu$.

\section{A spectral method in SPACE}

In this section we present a Galerkin spectral method for the space discretization of the fractional Cable equation. The use of the spectral method is motivated by

the memory feature of the fractional Cable equation. In the discrete version, this means that all previous solutions have to be saved to compute the solution at the current time level. In order to reduce the storage requirement, it is highly recommended to use the high-order method, such as the spectral method, for the space discretization, since higher-order methods require less degrees of freedom to achieve desired accuracy.

The Galerkin spectral discretization proceeds by approximating the solution by the polynomials of high degree. For spectral approximations in space of the problems (3.23), we define $\mathbb{P}_{N}(\Lambda)$ as the polynomials space of degree less than or equal to $N$ with respect to $x$. Let

$$
\mathbb{P}_{N}^{0}(\Lambda)=H_{0}^{1}(\Lambda) \cap \mathbb{P}_{N}(\Lambda) .
$$

We now consider the Galerkin spectral discretization to (3.23) as follows: find $u_{N}^{k+1} \in \mathbb{P}_{N}^{0}(\Lambda)$, such that for all $v_{N} \in \mathbb{P}_{N}^{0}(\Lambda)$

$$
\begin{aligned}
2\left(3 u_{N}^{k+1}\right. & \left.-4 u_{N}^{k}+u_{N}^{k-1}, v_{N}\right) \\
= & -\tilde{\alpha}\left[\left(u_{N}^{k+1}, v_{N}\right)-\sum_{j=0}^{k-1}\left(a_{j}-a_{j+1}\right)\left(u_{N}^{k-j}, v_{N}\right)-a_{k}\left(u_{N}^{0}, v_{N}\right)\right] \\
& -\tilde{\beta}\left[\left(\partial_{x} u_{N}^{k+1}, \partial_{x} v_{N}\right)-\sum_{j=0}^{k-1}\left(b_{j}-b_{j+1}\right)\left(\partial_{x} u_{N}^{k-j}, \partial_{x} v_{N}\right)-b_{k}\left(\partial_{x} u_{N}^{0}, \partial_{x} v_{N}\right)\right] \\
& -\tilde{\alpha}_{k+1}\left(u_{N}^{0}, v_{N}\right)-\tilde{\beta}_{k+1}\left(\partial_{x} u_{N}^{0}, \partial_{x} v_{N}\right), k \geq 1 .
\end{aligned}
$$


Then a simple rearrangement gives

$$
\begin{aligned}
& 2\left(3 u_{N}^{k+1}-4 u_{N}^{k}+u_{N}^{k-1}, v_{N}\right)+\tilde{\alpha}\left(u_{N}^{k+1}, v_{N}\right)+\tilde{\beta}\left(\partial_{x} u_{N}^{k+1}, \partial_{x} v_{N}\right) \\
& =\tilde{\alpha} \sum_{j=0}^{k-1}\left(a_{j}-a_{j+1}\right)\left(u_{N}^{k-j}, v_{N}\right)+\left(\tilde{\alpha} a_{k}-\tilde{\alpha}_{k+1}\right)\left(u_{N}^{0}, v_{N}\right) \\
& \quad+\tilde{\beta} \sum_{j=0}^{k-1}\left(b_{j}-b_{j+1}\right)\left(\partial_{x} u_{N}^{k-j}, \partial_{x} v_{N}\right) \\
& \quad+\left(\tilde{\beta} b_{k}-\tilde{\beta}_{k+1}\right)\left(\partial_{x} u_{N}^{0}, \partial_{x} v_{N}\right), k \geq 1 .
\end{aligned}
$$

To analyze the error of the space discretization, we define the $H^{1}$-orthogonal projection operator $\pi_{N}^{1}$ as follows: For all $\psi \in H_{0}^{1}(\Lambda)$, let $\pi_{N}^{1} \psi \in \mathbb{P}_{N}^{0}(\Lambda)$, such that

$$
\begin{aligned}
& 6\left(\pi_{N}^{1} \psi, v_{N}\right)+\tilde{\alpha}\left(\pi_{N}^{1} \psi, v_{N}\right)+\tilde{\beta}\left(\partial_{x} \pi_{N}^{1} \psi, \partial_{x} v_{N}\right) \\
& \quad=6\left(\psi, v_{N}\right)+\tilde{\alpha}\left(\psi, v_{N}\right)+\tilde{\beta}\left(\partial_{x} \psi, \partial_{x} v_{N}\right), \forall v_{N} \in \mathbb{P}_{N}^{0}(\Lambda) .
\end{aligned}
$$

Then the following projection estimate holds:

$$
\left\|\psi-\pi_{N}^{1} \psi\right\|_{1} \leq c N^{1-m}\|\psi\|_{m}, \quad \text { if } \psi \in H^{m}(\Lambda) \cap H_{0}^{1}(\Lambda), m \geq 1,
$$

where $\|\cdot\|_{1}$ is the modified $H^{1}$-norm defined in (3.20). This estimate can be proved by taking into account the standard projection error (see e.g. [3, 4]), and the norm equivalence.

To start the time iteration of (4.1), an initial condition will be needed for $u_{N}^{0}$. Here we take $u_{N}^{0}=\pi_{N}^{1} u^{0}$.

Theorem 4.1. Let $\left\{u^{k}\right\}_{k=1}^{K}$ be the solution of the semi-discrete problem (3.23) and $\left\{u_{N}^{k}\right\}_{k=1}^{K}$ the solution of the full-discrete problem (4.1) with the initial condition $u_{N}^{0}=\pi_{N}^{1} u^{0}$. If $u^{k} \in H^{m}(\Lambda), k \geq 0, m>1$, then the following error estimate holds

$$
\left\|u^{k}-u_{N}^{k}\right\|_{1} \leq c T \Delta t^{-1} N^{1-m} \max _{0 \leq j \leq k}\left\|u^{j}\right\|_{m}, k=0,1, \ldots, K
$$

Proof. Let $e_{N}^{k}=u^{k}-u_{N}^{k}, \tilde{e}_{N}^{k}=\pi_{N}^{1} u^{k}-u_{N}^{k}, \bar{e}_{N}^{k}=u^{k}-\pi_{N}^{1} u^{k}, k \geq 0$.

Similar to (4.1), we first rewrite the semi-discrete problem (3.23) under the form:

$$
\begin{aligned}
2\left(3 u^{k+1}-4 u^{k}+u^{k-1}, v\right)+\tilde{\alpha}\left(u^{k+1}, v\right)+\tilde{\beta}\left(\partial_{x} u^{k+1}, \partial_{x} v\right) \\
=\tilde{\alpha} \sum_{j=0}^{k-1}\left(a_{j}-a_{j+1}\right)\left(u^{k-j}, v\right)+\left(\tilde{\alpha} a_{k}-\tilde{\alpha}_{k+1}\right)\left(u^{0}, v\right) \\
\quad+\tilde{\beta} \sum_{j=0}^{k-1}\left(b_{j}-b_{j+1}\right)\left(\partial_{x} u^{k-j}, \partial_{x} v\right)+\left(\tilde{\beta} b_{k}-\tilde{\beta}_{k+1}\right)\left(\partial_{x} u^{0}, \partial_{x} v\right), \quad \forall v \in H_{0}^{1}(\Lambda) .
\end{aligned}
$$

Applying the projection operator $\pi_{N}^{1}$ to the above equation,and using (4.2) yields 


$$
\begin{aligned}
& 2\left(\pi_{N}^{1}\left(3 u^{k+1}-4 u^{k}+u^{k-1}\right), v_{N}\right) \\
& +\tilde{\alpha}\left(\pi_{N}^{1} u^{k+1}, v_{N}+\tilde{\beta}\left(\partial_{x} \pi_{N}^{1} u^{k+1}, \partial_{x} v_{N}\right)\right. \\
& =\tilde{\alpha} \sum_{j=0}^{k-1}\left(a_{j}-a_{j+1}\right)\left(\pi_{N}^{1} u^{k-j}, v_{N}\right)+\left(\tilde{\alpha} a_{k}-\tilde{\alpha}_{k+1}\right)\left(\pi_{N}^{1} u^{0}, v_{N}\right) \\
& \quad+\tilde{\beta} \sum_{j=0}^{k-1}\left(b_{j}-b_{j+1}\right)\left(\partial_{x} \pi_{N}^{1} u^{k-j}, \partial_{x} v_{N}\right) \\
& \quad+\left(\tilde{\beta} b_{k}-\tilde{\beta}_{k+1}\right)\left(\partial_{x} \pi_{N}^{1} u^{0}, \partial_{x} v_{N}\right)+e_{\pi}\left(v_{N}\right), \forall v_{N} \in \mathbb{P}_{N}^{0}(\Lambda),
\end{aligned}
$$

where $e_{\pi}\left(v_{N}\right)$ is associated to the projection error, defined by: $\forall v_{N} \in \mathbb{P}_{N}^{0}(\Lambda)$,

$$
\begin{aligned}
e_{\pi}\left(v_{N}\right)= & \left(8 \bar{e}_{N}^{k}-2 \bar{e}_{N}^{k-1}, v_{N}\right)+\tilde{\alpha} \sum_{j=0}^{k-1}\left(a_{j}-a_{j+1}\right)\left(\bar{e}_{N}^{k-j}, v_{N}\right)+\left(\tilde{\alpha} a_{k}-\tilde{\alpha}_{k+1}\right)\left(\bar{e}_{N}^{0}, v_{N}\right) \\
& +\tilde{\beta} \sum_{j=0}^{k-1}\left(b_{j}-b_{j+1}\right)\left(\partial_{x} \bar{e}_{N}^{k-j}, \partial_{x} v_{N}\right)+\left(\tilde{\beta} b_{k}-\tilde{\beta}_{k+1}\right)\left(\partial_{x} \bar{e}_{N}^{0}, \partial_{x} v_{N}\right) .
\end{aligned}
$$

By subtracting (4.1) from (4.6), then taking $v_{N}=\tilde{e}_{N}^{k+1}$, we obtain

$$
\begin{aligned}
& 2\left(3 \tilde{e}_{N}^{k+1}-4 \tilde{e}_{N}^{k}+\tilde{e}_{N}^{k-1}, \tilde{e}_{N}^{k+1}\right)+\tilde{\alpha}\left(\tilde{e}_{N}^{k+1}, \tilde{e}_{N}^{k+1}\right)+\tilde{\beta}\left(\partial_{x} \tilde{e}_{N}^{k+1}, \partial_{x} \tilde{e}_{N}^{k+1}\right) \\
& =\tilde{\alpha} \sum_{j=0}^{k-1}\left(a_{j}-a_{j+1}\right)\left(\tilde{e}_{N}^{k-j}, \tilde{e}_{N}^{k+1}\right)+\left(\tilde{\alpha} a_{k}-\tilde{\alpha}_{k+1}\right)\left(\tilde{e}_{N}^{0}, \tilde{e}_{N}^{k+1}\right) \\
& \quad+\tilde{\beta} \sum_{j=0}^{k-1}\left(b_{j}-b_{j+1}\right)\left(\partial_{x} \tilde{e}_{N}^{k-j}, \partial_{x} \tilde{e}_{N}^{k+1}\right)+\left(\tilde{\beta} b_{k}-\tilde{\beta}_{k+1}\right)\left(\partial_{x} \tilde{e}_{N}^{0}, \partial_{x} \tilde{e}_{N}^{k+1}\right) \\
& \quad+e_{\pi}\left(\tilde{e}_{N}^{k+1}\right) .
\end{aligned}
$$

Employing a similar technique as in the Theorem 3.2, we arrive at

$$
\begin{aligned}
& \left\|\tilde{e}_{N}^{k+1}\right\|_{0}^{2}+\left\|2 \tilde{e}_{N}^{k+1}-\tilde{e}_{N}^{k}\right\|_{0}^{2}-\left\|\tilde{e}_{N}^{k}\right\|_{0}^{2}-\left\|2 \tilde{e}_{N}^{k}-\tilde{e}_{N}^{k-1}\right\|_{0}^{2} \\
& +\left\|\tilde{e}_{N}^{k+1}-2 \tilde{e}_{N}^{k}+\tilde{e}_{N}^{k-1}\right\|_{0}^{2} \\
& \leq-\frac{\tilde{\alpha}}{2} \sum_{j=0}^{k+1} a_{j}\left\|\tilde{e}_{N}^{k+1-j}\right\|_{0}^{2}+\frac{\tilde{\alpha}}{2} \sum_{j=0}^{k} a_{j}\left\|\tilde{e}_{N}^{k-j}\right\|_{0}^{2}-\frac{\tilde{\beta}}{2} \sum_{j=0}^{k+1} b_{j}\left\|\partial_{x} \tilde{e}_{N}^{k+1-j}\right\|_{0}^{2} \\
& +\frac{\tilde{\beta}}{2} \sum_{j=0}^{k} b_{j}\left\|\partial_{x} \tilde{e}_{N}^{k-j}\right\|_{0}^{2}-\frac{\tilde{\alpha}_{k+1}}{2}\left\|\tilde{e}_{N}^{k+1}\right\|_{0}^{2}-\frac{\tilde{\beta}_{k+1}}{2}\left\|\partial_{x} \tilde{e}_{N}^{k+1}\right\|_{0}^{2}+\left|e_{\pi}\left(\tilde{e}_{N}^{k+1}\right)\right| .
\end{aligned}
$$


We are now led to estimate the term $\left|e_{\pi}\left(\tilde{e}_{N}^{k+1}\right)\right|$. First, by using Young's inequality to the first part of this term, we have

$$
\begin{aligned}
\mid & \left(8 \bar{e}_{N}^{k}-2 \bar{e}_{N}^{k-1}+\tilde{\alpha} \sum_{j=0}^{k-1}\left(a_{j}-a_{j+1}\right) \bar{e}_{N}^{k-j}+\left(\tilde{\alpha} a_{k}-\tilde{\alpha}_{k+1}\right) \bar{e}_{N}^{0}, \tilde{e}_{N}^{k+1}\right) \mid \\
\leq & \frac{1}{2 \tilde{\alpha}_{k+1}}\left\|8 \bar{e}_{N}^{k}-2 \bar{e}_{N}^{k-1}+\tilde{\alpha} \sum_{j=0}^{k-1}\left(a_{j}-a_{j+1}\right) \bar{e}_{N}^{k-j}+\left(\tilde{\alpha} a_{k}-\tilde{\alpha}_{k+1}\right) \bar{e}_{N}^{0}\right\|_{0}^{2} \\
& +\frac{\tilde{\alpha}_{k+1}}{2}\left\|\tilde{e}_{N}^{k+1}\right\|_{0}^{2} \\
\leq & \frac{1}{2 \tilde{\alpha}_{k+1}}\left[c+\tilde{\alpha}^{2} \sum_{j=0}^{k-1}\left(a_{j}-a_{j+1}\right)^{2}+\left(\tilde{\alpha} a_{k}-\tilde{\alpha}_{k+1}\right)^{2}\right] \max _{0 \leq j \leq k}\left\|\bar{e}_{N}^{j}\right\|_{0}^{2}+\frac{\tilde{\alpha}_{k+1}}{2}\left\|\tilde{e}_{N}^{k+1}\right\|_{0}^{2} \\
\leq & \frac{c}{\tilde{\alpha}_{k+1}}\left[1+\tilde{\alpha}^{2}\left(1-\alpha_{k+1}\right)^{2}\right] \max _{0 \leq j \leq k}\left\|\bar{e}_{N}^{j}\right\|_{0}^{2}+\frac{\tilde{\alpha}_{k+1}}{2}\left\|\tilde{e}_{N}^{k+1}\right\|_{0}^{2} \quad(\text { by Lemma } \underline{3.2}) \\
\leq & \frac{c}{\tilde{\alpha}_{k+1}}\left(1+\tilde{\alpha}^{2}\right) \max _{0 \leq j \leq k}\left\|\bar{e}_{N}^{j}\right\|_{0}^{2}+\frac{\tilde{\alpha}_{k+1}}{2}\left\|\tilde{e}_{N}^{k+1}\right\|_{0}^{2} .
\end{aligned}
$$

Similarly, we have

$$
\begin{aligned}
& \left|\left(\tilde{\beta} \sum_{j=0}^{k-1}\left(b_{j}-b_{j+1}\right) \partial_{x} \bar{e}_{N}^{k-j}+\left(\tilde{\beta} b_{k}-\tilde{\beta}_{k+1}\right) \partial_{x} \bar{e}_{N}^{0}, \partial_{x} \tilde{e}_{N}^{k+1}\right)\right| \\
& \leq \frac{1}{2 \tilde{\beta}_{k+1}}\left\|\tilde{\beta} \sum_{j=0}^{k-1}\left(b_{j}-b_{j+1}\right) \partial_{x} \bar{e}_{N}^{k-j}+\left(\tilde{\beta} b_{k}-\tilde{\beta}_{k+1}\right) \partial_{x} \bar{e}_{N}^{0}\right\|_{0}^{2} \\
& \quad+\frac{\tilde{\beta}_{k+1}}{2}\left\|\partial_{x} \tilde{e}_{N}^{k+1}\right\|_{0}^{2} \\
& \leq \frac{c}{\tilde{\beta}_{k+1}} \tilde{\beta}^{2} \max _{0 \leq j \leq k}\left\|\partial_{x} \bar{e}_{N}^{j}\right\|_{0}^{2}+\frac{\tilde{\beta}_{k+1}}{2}\left\|\partial_{x} \tilde{e}_{N}^{k+1}\right\|_{0}^{2} .
\end{aligned}
$$

Combining (4.8)-4.10) gives

$$
\begin{aligned}
& \left\|\tilde{e}_{N}^{k+1}\right\|_{0}^{2}+\left\|2 \tilde{e}_{N}^{k+1}-\tilde{e}_{N}^{k}\right\|_{0}^{2}-\left\|\tilde{e}_{N}^{k}\right\|_{0}^{2}-\left\|2 \tilde{e}_{N}^{k}-\tilde{e}_{N}^{k-1}\right\|_{0}^{2} \\
& \quad+\left\|\tilde{e}_{N}^{k+1}-2 \tilde{e}_{N}^{k}+\tilde{e}_{N}^{k-1}\right\|_{0}^{2} \\
& \leq-\frac{\tilde{\alpha}}{2} \sum_{j=0}^{k+1} a_{j}\left\|\tilde{e}_{N}^{k+1-j}\right\|_{0}^{2}+\frac{\tilde{\alpha}}{2} \sum_{j=0}^{k} a_{j}\left\|\tilde{e}_{N}^{k-j}\right\|_{0}^{2}-\frac{\tilde{\beta}}{2} \sum_{j=0}^{k+1} b_{j}\left\|\partial_{x} \tilde{e}_{N}^{k+1-j}\right\|_{0}^{2} \\
& \quad+\frac{\tilde{\beta}}{2} \sum_{j=0}^{k} b_{j}\left\|\partial_{x} \tilde{e}_{N}^{k-j}\right\|_{0}^{2} \\
& \quad+\frac{c}{\tilde{\alpha}_{k+1}}\left(1+\tilde{\alpha}^{2}\right) \max _{0 \leq j \leq k}\left\|\bar{e}_{N}^{j}\right\|_{0}^{2}+\frac{c}{\tilde{\beta}_{k+1}} \tilde{\beta}^{2} \max _{0 \leq j \leq k}\left\|\partial_{x} \bar{e}_{N}^{j}\right\|_{0}^{2} .
\end{aligned}
$$

By virtue of the definitions of $\tilde{\alpha}_{k+1}$ and $\tilde{\beta}_{k+1}$, i.e., (3.22), we have

$$
\frac{1}{\tilde{\alpha}_{k+1}} \leq c T^{\alpha} \triangle t^{-1}, \quad \frac{1}{\tilde{\beta}_{k+1}} \leq c T^{\beta} \triangle t^{-1} \text {. }
$$


Thus the last two terms in (4.11) can be bounded by

$$
\frac{c}{\tilde{\alpha}_{k+1}}\left(1+\tilde{\alpha}^{2}\right) \max _{0 \leq j \leq k}\left\|\bar{e}_{N}^{j}\right\|_{0}^{2}+\frac{c}{\tilde{\beta}_{k+1}} \tilde{\beta}^{2} \max _{0 \leq j \leq k}\left\|\partial_{x} \bar{e}_{N}^{j}\right\|_{0}^{2} \leq c T \triangle t^{-1} \max _{0 \leq j \leq k}\left\|\bar{e}_{N}^{j}\right\|_{1}^{2},
$$

where norm $\|\cdot\|_{1}$ is defined in (3.20). Now by letting

$$
\varepsilon_{N}^{k}:=\left\|\tilde{e}_{N}^{k}\right\|_{0}^{2}+\left\|2 \tilde{e}_{N}^{k}-\tilde{e}_{N}^{k-1}\right\|_{0}^{2}+\frac{\tilde{\alpha}}{2} \sum_{j=0}^{k} a_{j}\left\|\tilde{e}_{N}^{k-j}\right\|_{0}^{2}+\frac{\tilde{\beta}}{2} \sum_{j=0}^{k} b_{j}\left\|\partial_{x} \tilde{e}_{N}^{k-j}\right\|_{0}^{2},
$$

we obtain from (4.11),

$$
\varepsilon_{N}^{k+1} \leq \varepsilon_{N}^{k}+c T \triangle t^{-1} \max _{0 \leq j \leq k}\left\|\bar{e}_{N}^{j}\right\|_{1}^{2}, \forall k=0,1, \ldots, K .
$$

Consequently,

$$
\varepsilon_{N}^{k} \leq \varepsilon_{N}^{0}+c T k \triangle t^{-1} \max _{0 \leq j \leq k}\left\|\bar{e}_{N}^{j}\right\|_{1}^{2} \leq c T^{2} \triangle t^{-2} \max _{0 \leq j \leq k}\left\|\bar{e}_{N}^{j}\right\|_{1}^{2}, \forall k=0,1, \ldots, K .
$$

This implies that

$$
\left\|\tilde{e}_{N}^{k}\right\|_{1}^{2} \leq c T^{2} \triangle t^{-2} \max _{0 \leq j \leq k}\left\|\bar{e}_{N}^{j}\right\|_{1}^{2}, \forall k=0,1, \ldots, K .
$$

Finally, by using the triangle inequality, we obtain

$\left\|e_{N}^{k}\right\|_{1}=\left\|\tilde{e}_{N}^{k}+\bar{e}_{N}^{k}\right\|_{1} \leq\left\|\tilde{e}_{N}^{k}\right\|_{1}+\left\|\bar{e}_{N}^{k}\right\|_{1} \leq c T \triangle t^{-1} \max _{0 \leq j \leq k}\left\|\bar{e}_{N}^{j}\right\|_{1}, \quad \forall k=0,1, \ldots, K$.

This, together with (4.3), leads to (4.4).

Remark 4.1. In Theorem 4.1 the derived error estimate for the spatial approximation is affected by the inverse of the time step. However, our numerical tests given in section 5 show that this term is not really present in the numerical solutions. This disagreement probably means that the theoretical error estimate may be improved. In the actual estimation of the error at a given time level, the spatial errors at all previous time levels are simply accumulated due to the memory feature. The estimate could be improved by taking into account the fact that the coefficients $a_{j}$ and $b_{j}$ are decreasing as $j$ increases; that is, the memory effect is weaker for a longer time.

For the full-discrete error, we state the following result without giving detailed proof.

Theorem 4.2. Let $u$ be the solution of the continuous problem (2.3)-(2.5) and let $\left\{u_{N}^{k}\right\}_{k=1}^{K}$ be the solution of the full-discrete problem (4.1). If $u(\cdot, t) \in H^{m}(\Lambda), \forall t \in$ $[0, T], m>1$, then the following error estimate holds:

$$
\left\|u\left(\cdot, t_{k}\right)-u_{N}^{k}\right\|_{1} \leq c T\left(\Delta t^{\min (2-\alpha, 2-\beta)}+\Delta t^{-1} N^{1-m}\right), k=0,1, \ldots, K,
$$

where $c$ depends on $u$, but independent of $T, \triangle t$, and $N$. 


\section{Numerical VALidation}

5.1. Implementation. We start with some implementation details. As usual, in order to make problem (4.1) practical, all the integrals involved in (4.1) are evaluated by using suitable numerical quadratures. For the reason that the integrands in (4.1) are polynomials with respect to the space variable, we use the Gauss-LobattoLegendre (GLL) quadrature to compute the integrations.

Let's denote by $L_{N}(x)$ the Legendre polynomial of degree $N$. The points of the GLL quadrature formula, denoted by $x_{i}, i=0,1, \ldots, N$, are defined as the zeros of $\left(1-x^{2}\right) L_{N}^{\prime}(x)$. The associated weights, $\omega_{i}, i=0,1, \ldots, N$, are defined such that the following quadrature holds:

$$
\int_{-1}^{1} \varphi(x) d x=\sum_{i=0}^{N} \varphi\left(x_{i}\right) \omega_{i}, \quad \forall \varphi(x) \in \mathbb{P}_{2 N-1}(\Lambda) .
$$

Then we define the discrete inner product as follows:

$$
(\phi, \psi)_{N}=\sum_{i=0}^{N} \phi\left(x_{i}\right) \psi\left(x_{i}\right) \omega_{i}, \quad \forall \phi, \psi \in C^{0}(\bar{\Lambda}) .
$$

We now consider the discrete problem with numerical quadratures: Find $u_{N}^{k+1} \in$ $\mathbb{P}_{N}^{0}(\Lambda)$, such that for all $v_{N} \in \mathbb{P}_{N}^{0}(\Lambda)$,

$$
\begin{aligned}
& 6\left(u_{N}^{k+1}, v_{N}\right)_{N}+\tilde{\alpha}\left(u_{N}^{k+1}, v_{N}\right)_{N}+\tilde{\beta}\left(\partial_{x} u_{N}^{k+1}, \partial_{x} v_{N}\right)_{N} \\
& =\tilde{\alpha} \sum_{j=0}^{k-1}\left(a_{j}-a_{j+1}\right)\left(u_{N}^{k-j}, v_{N}\right)_{N}+\left(\tilde{\alpha} a_{k}-\tilde{\alpha}_{k+1}\right)\left(u_{N}^{0}, v_{N}\right)_{N} \\
& \quad+\tilde{\beta} \sum_{j=0}^{k-1}\left(b_{j}-b_{j+1}\right)\left(\partial_{x} u_{N}^{k-j}, \partial_{x} v_{N}\right)_{N}+\left(\tilde{\beta} b_{k}-\tilde{\beta}_{k+1}\right)\left(\partial_{x} u_{N}^{0}, \partial_{x} v_{N}\right)_{N} \\
& \quad+2\left(4 u_{N}^{k}-u_{N}^{k-1}, v_{N}\right)_{N}, k \geq 1 .
\end{aligned}
$$

For simplification, we will hereafter denote the RHS of (5.1) by $F\left(u_{N}^{0}, u_{N}^{1}, \ldots\right.$, $\left.u_{N}^{k} ; v_{N}\right)$.

To derive the linear system to be solved at each time level, we express the solution $u_{N}^{k+1}$ in terms of the Lagrangian interpolants based on the points $\left\{x_{i}\right\}_{i=0}^{N}$,

$$
u_{N}^{k+1}(x)=\sum_{j=0}^{N} u_{j}^{k+1} l_{j}(x),
$$

where $u_{j}^{k+1}=u_{N}^{k+1}\left(x_{j}\right)$ are the unknowns of the approximate solution, $l_{j}$ is the Lagrangian polynomial defined in $\Lambda$, i.e.,

$$
l_{j} \in \mathbb{P}_{N}(\Lambda), l_{j}\left(x_{i}\right)=\delta_{i j}, \forall i, j \in\{0,1, \ldots, N\},
$$

with $\delta_{i j}$ : the Kronecker-delta symbol.

By bringing (5.2) into (5.1), and taking into account the homogeneous boundary condition (i.e., $u_{0}^{k+1}=u_{N}^{k+1}=0$ ), then choosing the test function $v_{N}$ to be $l_{i}(x), i=$ 
$1,2, \ldots, N-1$, we obtain

$$
\begin{aligned}
(6+\tilde{\alpha}) & \left(\sum_{j=1}^{N-1} u_{j}^{k+1} l_{j}, l_{i}\right)_{N}+\tilde{\beta}\left(\sum_{j=1}^{N-1} u_{j}^{k+1} l_{j}^{\prime}, l_{i}^{\prime}\right)_{N} \\
= & F\left(u_{N}^{0}, u_{N}^{1}, \ldots, u_{N}^{k} ; l_{i}\right), i=1,2, \ldots, N-1 .
\end{aligned}
$$

where $l_{j}^{\prime}(x)=\partial_{x} l_{j}(x)$. Using the definition of $(\cdot, \cdot)_{N}$ to the above system gives

$$
\begin{aligned}
(6+\tilde{\alpha}) u_{i}^{k+1} \omega_{i}+\tilde{\beta} \sum_{j=1}^{N-1} u_{j}^{k+1} \sum_{q=0}^{N} l_{j}^{\prime}\left(x_{q}\right) l_{i}^{\prime}\left(x_{q}\right) \omega_{q} \\
=F\left(u_{N}^{0}, u_{N}^{1}, \ldots, u_{N}^{k} ; l_{i}\right), i=1,2, \ldots, N-1,
\end{aligned}
$$

or, in matrix form,

$$
\sum_{j=1}^{N-1} H_{i j} u_{j}^{k+1}=F_{i}, \quad i=1,2, \ldots, N-1,
$$

where $F_{i}=F\left(u_{N}^{0}, u_{N}^{1}, \ldots, u_{N}^{k} ; l_{i}\right)$, and for all $i, j \in\{0,1, \ldots, N\}$,

$$
\begin{aligned}
H_{i j}=(6+\tilde{\alpha}) B_{i j}+\tilde{\beta} A_{i j}, & B_{i j}=\omega_{i} \delta_{i j}, \\
A_{i j}=\sum_{q=0}^{N} D_{q i} D_{q j} \omega_{q}, & D_{i j}=l_{j}^{\prime}\left(x_{i}\right) .
\end{aligned}
$$

It is readily seen that the matrix $H$ is symmetric positive definite, thus we choose the conjugate gradient method to solve (5.3).

5.2. Numerical results. In this subsection, we present numerical results obtained by the proposed finite difference/spectral method to support our theoretical statements. The main purpose is to check the convergence behavior of the numerical solution with respect to the time step $\Delta t$ and polynomial degree $N$ used in the calculation.

In order to test the convergence rate of the proposed method, we will need an exact solution to evaluate the accuracy of the numerical solution. For the reason that the exact solution of the problem (2.3)-(2.5) is generally unavailable, we instead consider the Cable equation with a forcing term $f$ :

$$
\partial_{t} u=-{ }_{0} D_{t}^{\alpha} u+{ }_{0} D_{t}^{\beta} \partial_{x}^{2} u+f, \forall(x, t) \in \Lambda \times I .
$$

Then we consider the following exact solution:

$$
u(x, t)=t^{2} \sin (2 \pi x)
$$

It can be checked that the associated forcing term is

$$
f(x, t)=\left(2 t+\frac{\Gamma(3)}{\Gamma(3-\alpha)} t^{2-\alpha}+\frac{4 \pi^{2} \Gamma(3)}{\Gamma(3-\beta)} t^{2-\beta}\right) \sin (2 \pi x) .
$$

The corresponding Cable problem with suitable initial and boundary conditions is solved by the method presented in the previous sections. We compute the errors $\left\|u(T)-u_{N}^{K}\right\|$ in two discrete norms: $L^{2}$ and $H^{1}$. All the numerical results reported in the figures below have been evaluated at $T=1$. 


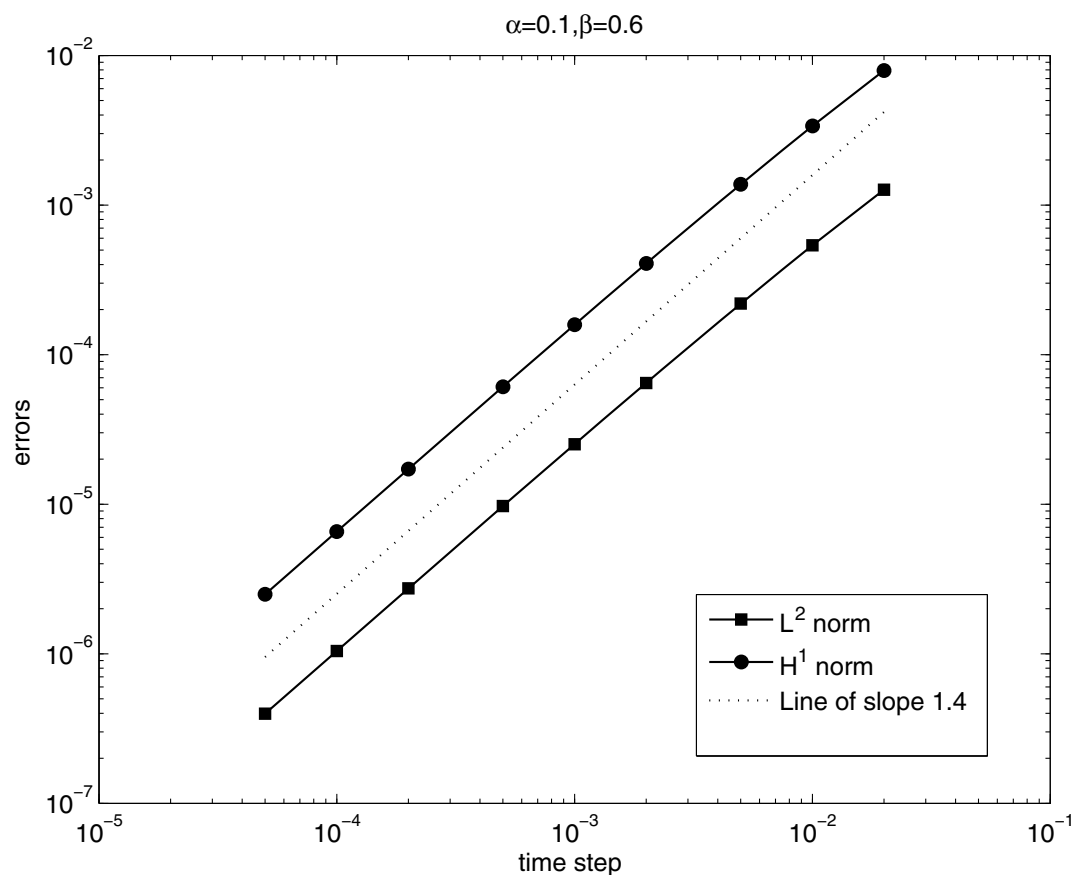

FiguRE 2. Errors as a function of the time step $\triangle t$ for $\alpha=0.1, \beta=$ 0.6 , and $N=16$.

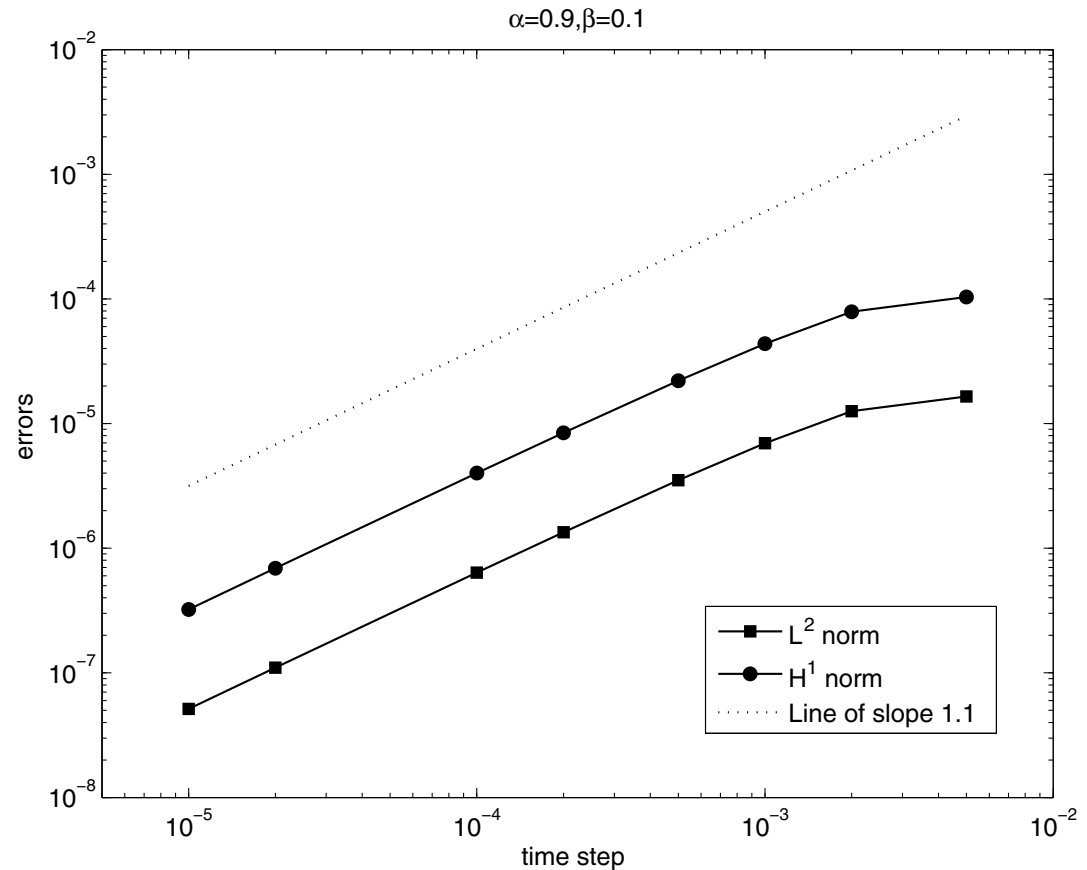

Figure 3. Errors as a function of the time step $\triangle t$ for $\alpha=0.9, \beta=$ 0.1 , and $N=16$. 


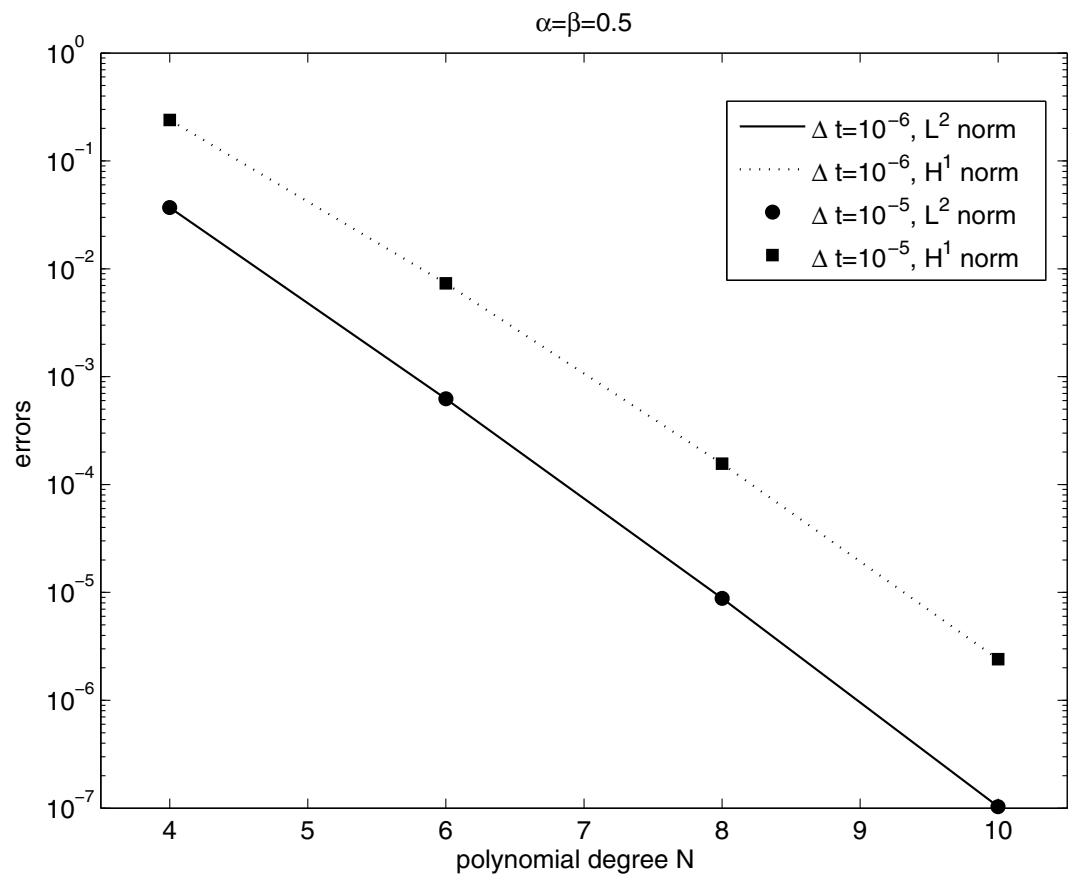

FiguRE 4. Errors as a function of the polynomial degree for $\alpha=$ $0.5, \beta=0.5$.

The first computational investigation is concerned with the temporal errors. In this first test, we fix $N=16$, a value large enough such that the space discretization error is negligible as compared with the time error. In Figures 2 and 3 , we plot the errors in the $L^{2}$ and $H^{1}$ semi-norms as a function of the time step sizes for two different sets of $\alpha$ and $\beta$. A logarithmic scale has been used for both $\triangle t$-axis and error-axis in these figures. As expected, the finite difference schema yields a fractional temporal approximation order $\min \{2-\alpha, 2-\beta\}$, that is, the slopes of the error curves in these $\log -\log$ plots are, respectively, 1.4 for $\alpha=0.1, \beta=0.6$ and 1.1 for $\alpha=0.9, \beta=0.1$.

Now we investigate the spatial error by letting $N$ vary and fixing the time step sufficiently small to avoid contamination of the temporal error. In Figure 4 we plot the errors as functions of $N$ for $\alpha=\beta=0.5$ by using two time step sizes $\Delta t=10^{-5}$ and $10^{-6}$. A logarithmic scale is now used for the error-axis. As predicted by the theoretical estimates, the errors show an exponential decay, since in these semi-log representation one observes that the error variations are essentially linear versus the degrees of polynomial. This is the so-called spectral accuracy, which can be expected for smooth solutions. Note that two different small enough time step sizes give exactly the same results indicating that the factor $\triangle t^{-1}$ in front of the spatial error estimate (see Theorems 4.1] and 4.2) is not really present in the numerical solution. In the future work, we will seek to improve the estimate by removing the factor $\Delta t^{-1}$. 


\section{Concluding Remarks}

We have presented a numerical method for the fractional Cable equation. The proposed method is based on a combination of some finite difference approaches in the time direction and a spectral method in the space direction. The convergence rate of the method is proven by providing a priori error estimate, and confirmed by a series of numerical tests. It is found that the combination of these finite difference schemes to the time derivatives results in a global $\min \{2-\alpha, 2-\beta\}$-order accuracy in time. This finding of the fractional order convergence rate is a generalization of the result obtained in 23. Concerning the spatial discretization, use of the spectral method leads to an exponential convergence in space. Some numerical experiments have been carried out to support the theoretical results.

In a future work, we plan to investigate the possibility to construct higher order schemes in time, as well as apply the present method to more realistic problems.

\section{APPENDIX}

Here we give a rigorous proof of (3.4). From (3.3), we have

$$
\begin{aligned}
r_{\alpha}^{k+1}= & \frac{1}{\Gamma(1-\alpha) \Delta t} \sum_{j=0}^{k}\left[-\int_{t_{j}}^{t_{j+1}} \partial_{\tau}^{2} u(x, \tau)\left(t_{j+1}-\tau\right) \int_{t_{j}}^{\tau} \frac{d s}{\left(t_{k+1}-s\right)^{\alpha}} d \tau\right. \\
& \left.-\int_{t_{j}}^{t_{j+1}} \partial_{\tau}^{2} u(x, \tau)\left(t_{j}-\tau\right) \int_{\tau}^{t_{j+1}} \frac{d s}{\left(t_{k+1}-s\right)^{\alpha}} d \tau\right] \\
= & \frac{1}{\Gamma(2-\alpha) \Delta t} \sum_{j=0}^{k} \int_{t_{j}}^{t_{j+1}} \partial_{\tau}^{2} u(x, \tau)\left[\left(t_{k+1}-\tau\right)^{1-\alpha} \Delta t\right. \\
& \left.-\left(t_{j+1}-\tau\right)\left(t_{k+1}-t_{j}\right)^{1-\alpha}+\left(t_{j}-\tau\right)\left(t_{k+1}-t_{j+1}\right)^{1-\alpha}\right] d \tau \\
= & \frac{1}{\Gamma(2-\alpha) \Delta t} \sum_{j=0}^{k} \int_{t_{j}}^{t_{j+1}} \partial_{\tau}^{2} u(x, \tau) R_{j}^{k+1}(\tau) d \tau .
\end{aligned}
$$

In the last equality of the above derivation we have used the notation $R_{j}^{k+1}(\tau)$ to denote

$R_{j}^{k+1}(\tau):=\left(t_{k+1}-\tau\right)^{1-\alpha} \Delta t-\left(t_{j+1}-\tau\right)\left(t_{k+1}-t_{j}\right)^{1-\alpha}+\left(t_{j}-\tau\right)\left(t_{k+1}-t_{j+1}\right)^{1-\alpha}$.

Now we are going to prove that $R_{j}^{k+1}(\tau)$ is nonnegative for all $\tau \in\left[t_{j}, t_{j+1}\right]$.

Let us first remark that

$$
R_{j}^{k+1}\left(t_{j}\right)=R_{j}^{k+1}\left(t_{j+1}\right)=0 .
$$

Moreover, a simple calculation shows:

$$
\partial_{\tau}^{2} R_{j}^{k+1}(\tau)=(1-\alpha)(-\alpha)\left(t_{k+1}-\tau\right)^{-1-\alpha} \Delta t \leq 0, \quad \text { for } \quad 0<\alpha<1 .
$$

As a consequence of a well-known result, we have

$$
R_{j}^{k+1}(\tau) \geq 0, \quad \text { for all } \tau \in\left[t_{j}, t_{j+1}\right] .
$$

Thus from equation (A.1), we obtain

$$
r_{\alpha}^{k+1} \leq \frac{M}{\Gamma(2-\alpha) \Delta t} \sum_{j=0}^{k} \int_{t_{j}}^{t_{j+1}} R_{j}^{k+1}(\tau) d \tau
$$


where $M=\max _{x \in \Lambda, \tau \in I} \partial_{\tau}^{2} u(x, \tau)$. The estimation of the error term $r_{\alpha}^{k+1}$ is then reduced to computing the integrals in the right-hand side:

$$
\begin{aligned}
\sum_{j=0}^{k} \int_{t_{j}}^{t_{j+1}} R_{j}^{k+1}(\tau) d \tau & \\
= & \sum_{j=0}^{k} \int_{t_{j}}^{t_{j+1}}\left[\left(t_{k+1}-\tau\right)^{1-\alpha} \Delta t-\left(t_{j+1}-\tau\right)\left(t_{k+1}-t_{j}\right)^{1-\alpha}\right. \\
& \left.\quad+\left(t_{j}-\tau\right)\left(t_{k+1}-t_{j+1}\right)^{1-\alpha}\right] d \tau
\end{aligned}
$$

Let

$$
s_{i}:=\frac{2}{2-\alpha}\left[(i+1)^{2-\alpha}-i^{2-\alpha}\right]-\left[(i+1)^{1-\alpha}+i^{1-\alpha}\right],
$$

then

$$
\begin{aligned}
\left|s_{i}\right|= & i^{1-\alpha}\left|\frac{2 i}{2-\alpha}\left(\left(1+\frac{1}{i}\right)^{2-\alpha}-1\right)-\left(1+\frac{1}{i}\right)^{1-\alpha}-1\right| \\
= & i^{1-\alpha} \mid \frac{2 i}{2-\alpha}\left(-1+1+(2-\alpha) \frac{1}{i}+\frac{(2-\alpha)(1-\alpha)}{2 !} \frac{1}{i^{2}}+\frac{(2-\alpha)(1-\alpha)(-\alpha)}{3 !} \frac{1}{i^{3}}\right. \\
& \left.+\frac{(2-\alpha)(1-\alpha)(-\alpha)(-\alpha-1)}{4 !} \frac{1}{i^{4}}+\cdots\right) \\
& -1-1-(1-\alpha) \frac{1}{i}-\frac{(1-\alpha)(-\alpha)}{2 !} \frac{1}{i^{2}}-\frac{(1-\alpha)(-\alpha)(-\alpha-1)}{3 !} \frac{1}{i^{3}}-\cdots \mid \\
= & i^{1-\alpha}\left|\left(\frac{1}{2 !}-\frac{2}{3 !}\right)(1-\alpha) \alpha \frac{1}{i^{2}}+\left(\frac{1}{3 !}-\frac{2}{4 !}\right)(1-\alpha) \alpha(-\alpha-1) \frac{1}{i^{3}}+\cdots\right| \\
\leq & i^{1-\alpha} \frac{1}{3 !}(1-\alpha) \alpha \frac{1}{i^{2}}\left(1+\frac{2(\alpha+1)}{4} \frac{1}{i}+\frac{3(\alpha+1)(\alpha+2)}{20} \frac{1}{i^{2}}+\cdots\right) \\
\leq & \frac{1}{3 !}(1-\alpha) \alpha \frac{1}{i^{1+\alpha}}\left(1+\frac{1}{i}+\frac{1}{i^{2}}+\cdots\right) \\
\leq & \frac{2}{3 !}(1-\alpha) \alpha \frac{1}{i^{1+\alpha}} \\
\leq & \frac{1}{i^{1+\alpha}} .
\end{aligned}
$$


Therefore, the series $\sum_{i=1}^{\infty} s_{i}$ converges for all $\alpha>0$. On the other side, a direct computation shows $s_{i}=0$ for $\alpha=0$ and $\alpha=1$. Consequently, there exists a positive constant $c$, which is independent of $\alpha$ and $k$, such that

$$
\sum_{i=0}^{k}\left\{\frac{2}{2-\alpha}\left[(i+1)^{2-\alpha}-k^{2-\alpha}\right]-\left[(i+1)^{1-\alpha}+i^{1-\alpha}\right]\right\} \leq c .
$$

As a result of the above estimates, we have

$$
r_{\alpha}^{k+1} \leq c \triangle t^{2-\alpha},
$$

where $c$ depends only on $M$, a constant measuring $\partial_{t}^{2} u$.

\section{REFERENCES}

1. F. Amblard, A. C. Maggs, B. Yurke, A. N. Pargellis, and S. Leibler. Subdiffusion and anomalous local viscoelasticity in actin networks. Phys. Rev. Lett., 77:4470, 1996.

2. E. Barkai, R. Metzler, and J. Klafter. From continuous time random walks to the fractional Fokker-Planck equation. Phys. Rev. E, 61:132-138, 2000. MR1736459(2000j:82035)

3. C. Bernardi and Y. Maday. Approximations spectrales de problemès aux limites elliptiques. Springer-Verlag, 1992. MR1208043 (94f:65112)

4. C. Bernardi and Y. Maday. Spectral methods. In P. G. Ciarlet and J. L. Lions, editors, Handbook of Numerical Analysis. Elsevier, Amsterdam, 1997. MR 1470226

5. J. Bouchaud and A. Georges. Anomalous diffusion in disordered media: Statistical mechanisms: models and physical applications. Phys. Rep., 195:127-293, 1990. MR1081295

6. E. Brown, E. Wu, W. Zipfel, and W. Webb. Measurement of molecular diffusion in solution by multiphoton fluorescence photobleaching recovery. Biophys. J., 77:2837-2849, 1999.

7. W. H. Deng. Finite element method for the space and time fractional Fokker-Planck equation. SIAM J. Numer. Anal., 47(1):204-226, 2008. MR2452858 (2009m:65175)

8. M. Dentz, A. Cortis, H. Scher, and B. Berkowitz. Time behaviour of solute in heterogeneous media: Transition from anomalous to normal transport. Adv. Water Resources, 27:155-173, 2004.

9. V. J. Ervin and J. P. Roop. Variational formulation for the stationary fractional advection dispersion equation. Numer. Methods Partial Differential Equations, 22:558-576, 2005. MR $2212226(2006 \mathrm{~m}: 65265)$

10. T. Feder, I. Brust-Mascher, J. Slattery, B. Baird, and W. Webb. Constrained diffusion or immobile fraction on cell surfaces: A new interpretation. Biophys. J., 70:2767-2773, 1996.

11. R. Ghosh. Mobility and clustering of individual low density lipoprotein receptor molecures on the surface of human skin fibroblasts. Ph.D. thesis. Cornell University, Ithaca, NY.

12. R. Ghosh and W. Webb. Automated detection and tracking of individual and clustered cell surface low density lipoprotein receptor molecures. Biophys. J., 66:1301-1318, 1994.

13. I. Goychuk, E. Heinsalu, M. Patriarca, G. Schmid, and Pänggi. Current and universal scaling in anomalous transport. Phys. Rev. E, 73:020101, 2006.

14. B. I. Henry, T. A. M. Langlands, and S. L. Wearne. Fractional cable models for spiny neuronal dendrites. Phys. Rev. Lett., 100(12):128103, 2008.

15. A. Hodgkin and A. Huxley. A quantitative description of membrane current and its application to conduction and excitation in nerve. J. Physiol., 117:500-544, 1952.

16. J. Jack, D. Noble, and R. Tsien. Electrical current flow in excitable cells. Oxford University Press, Oxford, 1975.

17. D. Junge. Nerve and Muscle Excitation (2nd edition ed.). Sinauer Associates, Inc., Sunderland, Massachusetts, 1981.

18. C. Koch. Biophysics of Computation, Information Processing in Single neurons, Computational Neuroscience. Oxford University Press, New York, 1999.

19. A. Kusumi, C. Nakada, K. Ritchie, K. Murase, K. Suzuki, H. Murakoshi, R. Kasai, J. Kondo, and T. Fujiwara. Paradigm shift of the plasma membrane concept from two-dimensional continuum fluid to the partitioned fluid: Highspeed single-molecure tracking of membrane molecures. Annu. Rev. Biophys. Biomol. Struct., 34:351-378, 2005. 
20. T. A. M. Langlands and B. I. Henry. The accuracy and stability of an implicit solution method for the fractional diffusion equation. J. Comput. Phys., 205(2):719-736, 2005. MR 2135000 (2005m:65174)

21. E. K. Lenzi, R. S. Mendes, K. S. Fa, and L. C. Malacarne. Anomalous diffusion: Fractional Fokker-Planck equation and its solutions. J. Math. Phys., 44:2179-2185, 2003. MR 1972771 (2004f:82060)

22. Xianjuan Li and Chuanju Xu. A space-time spectral method for the time fractional diffusion equation. SIAM J. Numerical Analysis, 47(3):2108-2131, 2009. MR 2519596

23. Y. M. Lin and C. J. Xu. Finite difference/spectral approximation for the time fractional diffusion equations. J. Comput. Phys., 2(3):1533-1552, 2007. MR2349193 (2008i:65200)

24. F. Mainardi. Fractional diffusive waves in viscoelastic solids. Nonlinear Waves in Solids, pages 93-97, 1995.

25. R. Metzler and J. Klafter. The random walk's guide to anomalous diffusion: A fractional dynamics approach. Phys. Rep., 339:1-77, 2000. MR1809268(2001k:82082)

26. R. Metzler, J. Klafter, and I. Sokolov. Anomalous transport in external fields: Continuous time random walks and fractional diffusion equations extended. Phys. Rev. E, 58:1621-1633, 1998.

27. H. P. Müller, R. Kimmich, and J. Weis. NMR flow velocity mapping in random percolation model objects: Evidence for a power-law dependence of the volume-averaged velocity on the probe-volume radius. Phys. Rev. E, 54:5278-5285, 1996.

28. I. Podlubny. Fractional Differential Equations. Academic Press, San Diego,, 1999. MR1658022 (99m:26009)

29. N. Qian and T. Sejnowski. An electro-diffusion model for computing membrane potentials and ionic concentrations in branching dendrites, spines and axons. Biol. Cybern., 62:1-15, 1989.

30. W. Rall. Branching dendritic trees and motoneuron membrane resistivity. volume 1, pages 491-527. 1959.

31. W. Rall. Core conductor theory and cable properties of neurons. In R. Poeter, editor, Handbook of Physiology: The Nervous System, Vol. 1 (Chapter 3), pages 39-97. American Physiological Society, Bethesda, MD, 1977.

32. K. Ritchie. Detection of non-Browian diffusion in the cell membrane in single molecure tracking. Biophys. J., 88:2266-2277, 2005.

33. H. Scher and M. Lax. Stochastic transport in a disordered solid. Phys. Rev. B, 7:4491-4502, 1973. MR0391854 (52:12673)

34. H. Scher and E. Montroll. Anomalous transit-time dispersion in amorphous solids. Phys. Rev. $B, 12: 2455-2477,1975$.

35. I. Segev, J. Fleshman, and R. Burke. Compartmental models of complex neurons. In C. Koch and I. Segev, editors, Methods in Neuronal Modelling. MIT Press, Cambridge, MA

36. R. Simson, B. Yang, S. Moore, P. Doherty, F. Walsh, and K. Jacobson. Structural mosaicism on the submicron scale in the plasma membrane. Biophys. J., 74:297-308, 1998

37. P. Smith, I. Morrison, K. Wilson, N. Fernandez, and R. Cherry. Constrained diffusion or immobile fraction on cell surfaces: A new interpretation. Biophys. J., 76:3331-3344, 1999.

38. M. Wachsmuth, T. Weidemann, G. Mǔller, U. Hoffmann-Rohrer, T.Knoch, W. Waldeck, and J. Langowski. Analyzing intracellular binding and diffusion with continuous fluorescence photobleaching. Biophys. J., 84:3353-3363, 2003.

39. E. R. Weeks. Experimental studies of anomalous diffusion, blocking phenomena, and twodimensional turbulence. Ph.D. thesis. University of Texas at Austin.

40. W. Wyss. The fractional diffusion equation. J. Math. Phys., 27(11):2782-2785, 1986. MR861345 (87m:44008)

School of Mathematical Sciences, Xiamen University, 361005 Xiamen, China

School of Mathematical Sciences, Xiamen University, 361005 Xiamen, China

School of Mathematical Sciences, Xiamen University, 361005 Xiamen, China

E-mail address: cjxu@xmu.edu.cn

URL: http://1sec.cc.ac.cn/ ${ }^{\sim} \mathrm{cj} \mathrm{xu}$ 